This item was submitted to Loughborough's Research Repository by the author.

Items in Figshare are protected by copyright, with all rights reserved, unless otherwise indicated.

\title{
Topology and bifurcations in nonholonomic mechanics
}

PLEASE CITE THE PUBLISHED VERSION

http://dx.doi.org/10.1142/S0218127415300281

\section{PUBLISHER}

(c) World Scientific Publishing Company

\section{VERSION}

SMUR (Submitted Manuscript Under Review)

\section{PUBLISHER STATEMENT}

This work is made available according to the conditions of the Creative Commons Attribution-NonCommercialNoDerivatives 4.0 International (CC BY-NC-ND 4.0) licence. Full details of this licence are available at: https://creativecommons.org/licenses/by-nc-nd/4.0/

\section{LICENCE}

CC BY-NC-ND 4.0

\section{REPOSITORY RECORD}

Bizyaev, Ivan A., Alexey V. Bolsinov, Alexey V. Borisov, and Ivan S. Mamaev. 2019. "Topology and Bifurcations in Nonholonomic Mechanics". figshare. https://hdl.handle.net/2134/19383. 


\title{
TOPOLOGY AND BIFURCATIONS IN NONHOLONOMIC MECHANICS
}

\author{
IVAN MAMAEV \\ Udmurt State University, Universitetskaya 1, \\ Izhevsk, 426034, Russia \\ mamaev@rcd.ru \\ ALEXEY BOLSINOV \\ School of Mathematics, Loughborough University \\ United Kingdom, LE11 3TU, Loughborough, Leicestershire \\ A.Bolsinov@lboro.ac.uk \\ IVAN BIZYAEV \\ Udmurt State University, Universitetskaya 1, \\ Izhevsk, 426034, Russia \\ bizaev_90@mail.ru
}

Received (to be inserted by publisher)

\begin{abstract}
This paper develops topological methods for qualitative analysis of the behavior of nonholonomic dynamical systems. Their application is illustrated by considering a new integrable system of nonholonomic mechanics, called a nonholonomic hinge. Although this system is nonholonomic, it can be represented in Hamiltonian form with a Lie-Poisson bracket of rank 2. This LiePoisson bracket is used to perform stability analysis of fixed points. In addition, all possible types of integral manifolds are found and a classification of trajectories on them is presented.
\end{abstract}

Keywords: nonholonomic hinge, topology, bifurcation diagram, tensor invariants, Poisson bracket, stability

\section{Introduction}

The paper deals with a new integrable problem of nonholonomic mechanics. A physical treatment of the system is given in [Kharlamov \& Kharlamov, 1995], and the system itself is called a nonholonomic hinge, which consists of two coupled rigid bodies connected via a rotating wheel (similar to Wagners interpretation [Wagner, 1941] of the Suslov problem, in the Suslov problem, one of the bodies is fixed in space, while in a nonholonomic hinge both bodies are free). More general statements of the problem of a nonholonomic hinge, as well as other integrable cases, are presented in [Bizyaev et al., 2013], where possible applied systems for the use of the nonholonomic hinge in engineering are also mentioned.

The problem of a nonholonomic hinge is of great interest for studying the topology of invariant manifolds and the stability of critical trajectories (fixed points). Compared to the classical problems of nonholonomic mechanics (the Chaplygin ball, the dynamics of a disk), the topology of this system is quite unusual and requires special consideration. Note that the nonholonomic Suslov problem also differs from 
the standard Liouville situation, and the motion on common level sets of first integrals occurs not in quasiperiodic orbits of tori, but on a two-dimensional manifold of genus greater than 1. For a recent analysis of the Suslov problem, see [Borisov et al., 2010].

This paper develops a series of the authors studies in the topology and stability of integrable systems [Bolsinov et al., 2010], Poisson geometry, and the hierarchy of dynamical behavior of nonholonomic systems [Borisov \& Mamaev, 2002, 2008; Borisov et al., 2002, 2013]. A peculiarity of the integrable problem we are considering is that the mechanism of its integrability is not standard. Though this problem is nonholonomic, it turns out that it can be presented in Hamiltonian form with a Lie-Poisson bracket of rank two. It is generally accepted that such systems have the following properties:

- Liouville integrability: symplectic leaves are two-dimensional and a single first integral, a Hamiltonian, is sufficient for integrability;

- after restriction to a symplectic leaf, we get a one-degree-of-freedom Hamiltonian system with its trajectories coinciding with the Hamiltonian level curves, while trajectories of a more complex structure such as irrational orbits of a torus are absent;

- bounded trajectories of the system are closed except for those that asymptotically approach the equilibrium position.

It is well known that the absence of globally defined Casimir functions for rank 2 brackets may lead to nonintegrability and even to chaos. In the problem that we are considering, the situation is intermediate. Although such a set of global Casimir functions does not exist, it is first integrated by quadratures. However, the described behavior pattern of the trajectories is no longer realized. Note that another class of rank 2 brackets that does not lead to integrability is mentioned in [Borisov et al., 2010]. We also define a special class of rank 2 brackets for which statements about integrability hold. Note that this integration mechanism has not been considered before, and the classical Liouville theorem is not applicable in this case.

In this paper, we present the general results concerning the stability of fixed points using Poisson brackets of rank 2 (the general criterion is given in the Appendix). Graphs of stability regions for the problem under consideration are built. A topological analysis of integral manifolds is performed and a classification of trajectories on these manifolds is provided. A three-dimensional bifurcation diagram is built in the space of values of first integrals. The last section of the paper discusses the absolute dynamics of a hinge associated with the description of the orientation of the inner body and the shell. It is shown that for some particularly remarkable motions corresponding to fixed points of the reduced system, the bundle of bodies executes double-period motion in the absolute space, while the nonsingular trajectories in the phase space are periodic and quasiperiodic orbits of two-dimensional tori.

Despite the fact that the paper deals with a specific problem of a nonholonomic hinge, its research methods are quite general. This problem-to-method approach is unfortunately rarely seen in the current literature on nonholonomic systems. Some studies formulate somewhat strange results concerning general nonholonomic systems, commonly using the simplest problems as examples (such as the rolling of an inhomogeneous ball), where everything is quite clear as it is (this comment relates to the issues of reduction, the existence of linear integrals, nonholonomic integrators, etc.). Nonholonomic mechanics cannot be developed without considering increasingly complex problems (a special term, "hierarchy of dynamic behavior", was introduced for this purpose in [Borisov \& Mamaev, 2002, 2008; Borisov et al., 2002, 2013]) and without refining special methods for investigating such systems while solving them. This problem demonstrates absolutely new topological and dynamic features that have never been encountered before, and the solution of this problem using the methods of Hamiltonian mechanics and Poisson geometry has given insight into new aspects of the behavior of nonholonomic systems.

\section{Realization of Constraints and Equations of Motion}

G. K. Suslov (see [Suslov, 1946, p. 325]) considered a system consisting of two bodies, each of which rotates about a fixed point, and which are connected with each other in such a way that the (nonholonomic) constraint is satisfied:

$$
(\boldsymbol{e}, \boldsymbol{\omega})+(\boldsymbol{E}, \boldsymbol{\Omega})=0,
$$


where $\boldsymbol{\omega}, \boldsymbol{\Omega}$ are the angular velocity vectors of the bodies, and $\boldsymbol{e}, \boldsymbol{E}$ are unit vectors rigidly attached to each of the bodies. He assumed that this constraint can be realized by means of "a very long, elastic, torsion-free thread", Fig. 1. Such a realization is incorrect, since it is well known that the rotation of the thread through a nonzero angle can arise not due to its torsions but due to a change in shape [Borisov et al., 2010; Fuller, 1971]. A correct (from the theoretical point of view) realization of this type of constraint for one body with a fixed point was proposed by V. Wagner [Wagner, 1941]. Later, a similar realization was also pointed out in [Kharlamov \& Kharlamov, 1995] and was called by the authors a nonholonomic hinge.

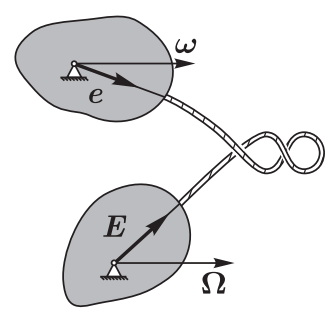

Fig. 1.

In this paper, we consider the problem of free motion of a bundle of two bodies connected via a nonholonomic hinge. The outer body is a spherical shell, with a rigid body moving inside and connected with it by means of sharp wheels in such a manner that relative rotations about the vector $\boldsymbol{e}$ fixed in the inner body are excluded (Fig. 2):

$$
(\boldsymbol{\omega}-\boldsymbol{\Omega}, \boldsymbol{e})=0,
$$

where $\boldsymbol{\Omega}, \boldsymbol{\omega}$ are the angular velocities of the shell and the inner body, respectively. In order to prohibit relative rotations of the bodies only along one direction, the points of contact of the wheels with the inner surface of the shell must lie on one straight line passing through the center of the sphere $C$ (Fig. 2). The arising constraint (2) is a particular case of the Suslov constraint (1). Furthermore, we shall assume that the centers of mass of the shell and the body coincide and are at the geometrical center of the sphere $C$.

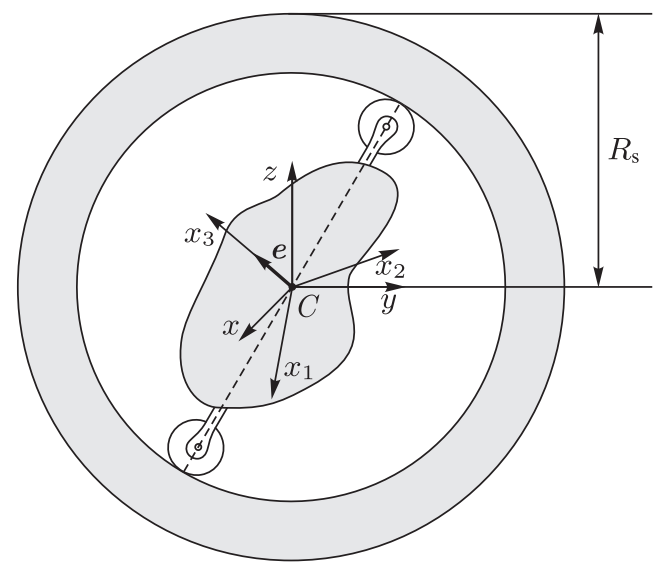

Fig. 2. A dynamically symmetrical spherical shell with a nonholonomic hinge inside.

We choose a moving coordinate system $C x_{1} x_{2} x_{3}$, rigidly attached to the inner body in such a manner that $C x_{3} \| \boldsymbol{e}$. Then the constraint equation becomes

$$
f_{0}=\omega_{3}-\Omega_{3}=0 \quad \text { (the Suslov constraint). }
$$


We shall assume that the tensor of inertia of the shell, $I_{\mathrm{s}} \mathbf{E}$, is spherical (here and in the sequel, the index s denotes the shell); moreover, in this paper we shall restrict ourselves to the case where the vector $\boldsymbol{e}$ coincides with the direction of one of the principal axes of inertia of the inner body. The kinetic energy of the entire system can be represented as

$$
T=\frac{1}{2}\left(I_{\mathrm{s}} \boldsymbol{\Omega}^{2}+(\boldsymbol{\omega}, \mathbf{I} \boldsymbol{\omega})\right),
$$

where $\mathbf{I}=\operatorname{diag}\left(I_{1}, I_{2}, I_{3}\right)$ is the tensor of inertia of the inner body (the axes $C x_{1} x_{2} x_{3}$ are assumed here to be the principal axes of inertia).

Using the formalism of [Borisov \& Mamaev, 2008], the equations of motion in the moving coordinate system rigidly attached to the inner body can be written explicitly as

$$
I_{\mathrm{S}} \dot{\boldsymbol{\Omega}}+I_{\mathrm{S}} \boldsymbol{\omega} \times \boldsymbol{\Omega}=-\lambda_{0} \boldsymbol{e}, \quad \mathbf{I} \dot{\boldsymbol{\omega}}+\boldsymbol{\omega} \times \mathbf{I} \boldsymbol{\omega}=\lambda_{0} \boldsymbol{e},
$$

where $\lambda_{0}$ is the undetermined multiplier and $\boldsymbol{e}=(0,0,1)$. Eliminating $\lambda_{0}$ using the constraint equation (3) and simplifying, we obtain the following closed system:

$$
\begin{gathered}
\dot{\Omega}_{1}=\omega_{3}\left(\Omega_{2}-\omega_{2}\right), \quad \dot{\Omega}_{2}=\omega_{3}\left(\omega_{1}-\Omega_{1}\right), \\
I_{1} \dot{\omega}_{1}=\left(I_{2}-I_{3}\right) \omega_{2} \omega_{3}, \quad I_{2} \dot{\omega}_{2}=\left(I_{3}-I_{1}\right) \omega_{1} \omega_{3}, \\
\left(I_{\mathrm{s}}+I_{3}\right) \dot{\omega}_{3}=I_{\mathrm{s}}\left(\Omega_{1} \omega_{2}-\Omega_{2} \omega_{1}\right)+\left(I_{1}-I_{2}\right) \omega_{1} \omega_{2} .
\end{gathered}
$$

These equations need to be supplemented with kinematic relations allowing one to determine the orientation of the shell and the inner body from given angular velocities $\boldsymbol{\omega}(t)$ and $\boldsymbol{\Omega}(t)$. For brevity, we shall refer to it as reconstruction of absolute dynamics. This issue is discussed in more detail in Section 6 . We shall call equations (5) a reduced system and consider them in more detail.

Note that since the entire system is not under the influence of any fields, its center of mass (which coincides with the geometric center of the shell) can be taken to be fixed relative to a certain inertial coordinate system.

\section{Tensor Invariants of the Reduced System}

In order to analyze the dynamics of this system, let us first consider what tensor invariants [Borisov \& Mamaev, 2002, 2008; Borisov et al., 2002, 2013] it possesses. First, the system (5) admits three quadratic first integrals

$$
\begin{gathered}
E=\frac{1}{2} I_{\mathrm{s}}\left(\Omega_{1}^{2}+\Omega_{2}^{2}\right)+\frac{1}{2}\left(I_{1} \omega_{1}^{2}+I_{2} \omega_{2}^{2}+\left(I_{3}+I_{\mathrm{s}}\right) \omega_{3}^{2}\right), \\
C_{1}=I_{1}\left(I_{1}-I_{3}\right) \omega_{1}^{2}+I_{2}\left(I_{2}-I_{3}\right) \omega_{2}^{2}, \quad C_{2}=\left(I_{1} \omega_{1}-I_{3} \Omega_{1}\right)^{2}+\left(I_{2} \omega_{2}-I_{3} \Omega_{2}\right)^{2} .
\end{gathered}
$$

Here, the first of the integrals is the kinetic energy of the system and its preservation follows from the general theorems of dynamics [Borisov \& Mamaev, 2008; Kozlov \& Kolesnikov, 1978], and the other two integrals are easy to find if one notices that the first four equations of the system (5), after division by $\omega_{3}$, form a closed linear system.

Moreover, equations (5) preserve the standard invariant measure:

$$
\mu=d \Omega_{1} d \Omega_{2} d \omega_{1} d \omega_{2} d \omega_{3} .
$$

It turns out that equations (5) preserve another tensor invariant, a Poisson structure, and can therefore be written in Hamiltonian form.

Proposition 1. The equations of motion (5) can be represented in the Hamiltonian form

$$
\begin{gathered}
\dot{\omega}_{i}=\left\{\omega_{i}, H\right\}, \quad \dot{\Omega}_{j}=\left\{\Omega_{j}, H\right\} \\
H=\frac{1}{2} \omega_{3}^{2}+\frac{1}{2} \frac{I_{1} \omega_{1}^{2}+I_{2} \omega_{2}^{2}+I_{s}\left(\Omega_{1}^{2}+\Omega_{2}^{2}\right)}{I_{s}+I_{3}},
\end{gathered}
$$


with the degenerate Lie-Poisson bracket of rank two

$$
\begin{gathered}
\left\{\Omega_{1}, \omega_{3}\right\}=\Omega_{2}-\omega_{2}, \quad\left\{\Omega_{2}, \omega_{3}\right\}=\omega_{1}-\Omega_{1}, \\
\left\{\omega_{1}, \omega_{3}\right\}=\frac{I_{2}-I_{3}}{I_{1}} \omega_{2}, \quad\left\{\omega_{2}, \omega_{3}\right\}=\frac{I_{3}-I_{1}}{I_{2}} \omega_{1} .
\end{gathered}
$$

Proof. Proof is a straightforward calculation of the equations and the Jacobi identity.

Using the variables $\boldsymbol{z}=\left(\Omega_{1}, \Omega_{2}, \omega_{1}, \omega_{2}, \omega_{3}\right)$, the Poisson structure (8) can be written as a matrix

$$
\mathbf{J}=\left(\begin{array}{ccccc}
0 & 0 & 0 & 0 & \Omega_{2}-\omega_{2} \\
0 & 0 & 0 & 0 & -\left(\Omega_{1}-\omega_{1}\right) \\
0 & 0 & 0 & 0 & \frac{I_{2}-I_{3}}{I_{1}} \omega_{2} \\
0 & 0 & 0 & 0 & -\frac{I_{1}-I_{3}}{I_{2}} \omega_{1} \\
* & * & * & * & 0
\end{array}\right),
$$

where the asterisks denote the nonzero elements of the matrix obtained from the condition of skewsymmetry $\mathbf{J}^{\top}=-\mathbf{J}$.

It is easy to verify that the integrals $C_{1}, C_{2}(6)$ are Casimir functions of this Poisson structure, and the third Casimir function in the general case is not globally defined.

As will be shown below, the Hamiltonian representation (7), (8) in this case is a fundamental property of the reduced system (5) and can be used not only to obtain integrals of motion and an invariant measure but also to integrate the system by quadratures and to make a substantial progress in its qualitative analysis.

\section{Poisson Structures of Rank Two and Integrability by Quadratures}

\subsection{General Properties of Poisson Structures of Type (8)}

Linear Poisson structures of type (8) are interesting in many respects. They have appeared in various contexts and studied, for example, in [Bolsinov \& Taimanov, 2000a,b; Konyaev, 2014; Butler, 2003]. Their main property is that they are of rank two ${ }^{1}$. Thus, their symplectic leaves are two-dimensional, Hamiltonian systems (after restriction to a symplectic leaf) have one degree of freedom and therefore, at first glance, should be automatically Liouville integrable for any choice of the Hamiltonian. The problem, however, lies in the fact that not all Casimir functions of such Poisson brackets can be defined globally. In this subsection, we discuss this effect in more detail and at the same time point out some general properties of the brackets (8).

First of all, we give a description of the 5-dimensional Lie algebra $\mathfrak{g}$ corresponding to the bracket (8) that will be convenient for further investigation. It is easy to see that $\mathfrak{g}$ can be written as the semidirect sum of a one-dimensional Lie algebra and a four-dimensional space $\mathbb{R}^{4}$ which is considered as a commutative subalgebra. The matrix representation of this Lie algebra is as follows:

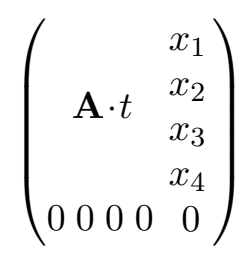

where $\mathbf{A}=\left(a_{i}^{j}\right)$ is a certain fixed $4 \times 4$ matrix and $x_{1}, x_{2}, x_{3}, x_{4}, t$ are considered as independent parameters. In our particular case, the variables $\Omega_{1}, \Omega_{2}, \omega_{1}, \omega_{2}, \omega_{3}$ correspond to (more precisely, are dual

\footnotetext{
${ }^{1}$ All Poisson brackets of rank two are described in a recent paper by A. Yu. Konyaev [Konyaev, 2014]. The issue of classification of such brackets was raised in [Bolsinov et al., 2012], where the following question was also formulated: "Are there any Hamiltonian systems with brackets that do not admit globally defined independent $n-1$ integrals?". It is the system under consideration that yields a positive answer to that question.
} 
to) the parameters $x_{1}, x_{2}, x_{3}, x_{4}, t$, respectively, and the matrix $\mathbf{A}$ has the form

$$
\mathbf{A}=\left(\begin{array}{cccc}
0 & 1 & 0 & 0 \\
-1 & 0 & 0 & 0 \\
0 & -1 & 0 & \frac{I_{1}-I_{3}}{I_{2}} \\
1 & 0 & \frac{I_{3}-I_{2}}{I_{1}} & 0
\end{array}\right)
$$

Now let us make some general comments about the linear Poisson brackets of this type. In other words, we assume for now that the matrix $\mathbf{A}$ is completely arbitrary, and therefore instead of $\left(x_{1}, \ldots, x_{4}\right)$ we consider the $n$-dimensional vector $\boldsymbol{x} \in \mathbb{R}^{n}$. The following statement summarizes the basic properties of Lie algebras of the type under consideration and the related Poisson brackets, which can be useful for applications (see also [Konyaev, 2014]).

Proposition 2. Let $\mathbf{A}=\left(a_{i}^{j}\right)$ be a nonzero $n \times n$ matrix. Consider an $n+1$-dimensional matrix Lie algebra of the form

$$
\mathfrak{g}=\left\{\left(\begin{array}{cc}
\mathbf{A} \cdot t & \boldsymbol{x} \\
0 & 0
\end{array}\right), \quad t \in \mathbb{R}, \boldsymbol{x} \in \mathbb{R}^{n}\right\}
$$

Let $y_{1}, \ldots, y_{n}, \tau$ denote the coordinates on the dual space $\mathfrak{g}^{*}$, dual to the coordinates $x_{1}, \ldots, x_{n}, t$ on $\mathfrak{g}$.

(1) Lie algebras of this type corresponding to the conjugate matrices $\mathbf{A}$ and $\mathbf{A}^{\prime}=\mathbf{C A C} \mathbf{C}^{-1}$ are isomorphic.

(2) The Poisson-Lie bracket on $\mathfrak{g}^{*}$ is of rank 2.

(3) A Lie algebra $\mathfrak{g}$ is unimodular if and only if $\operatorname{Tr} \mathbf{A}=0$. In this case, any Hamiltonian vector field on $\mathfrak{g}^{*}$ has a natural invariant measure $d y_{1} \ldots d y_{n} d \tau$.

(4) The Poisson tensor $\mathbf{J}$ corresponding to a Lie algebra $\mathfrak{g}$ can be presented as a decomposable bivector $\boldsymbol{\eta} \wedge \boldsymbol{\xi}^{\prime}$ where $\boldsymbol{\eta}$ and $\boldsymbol{\xi}^{\prime}$ are smooth vector fields on $\mathfrak{g}^{*}$. Namely, $\boldsymbol{\eta}=\partial_{\tau}$ is the constant vector field and $\boldsymbol{\xi}$ is a natural extension of the linear vector field $\boldsymbol{\xi}$ on $\mathbb{R}^{n}(\boldsymbol{y})$ to $\mathfrak{g}^{*}=\mathbb{R}^{n}(\boldsymbol{y}) \times \mathbb{R}^{1}(\tau)$. In the coordinates $y_{1}, \ldots, y_{n}$ this vector field has the form of $\sum a_{i}^{j} y_{i} \partial_{y_{j}}$. In matrix form:

$$
\mathbf{J}=\left(\begin{array}{cc}
0 & \mathbf{A}^{\top} \boldsymbol{y} \\
-\boldsymbol{y}^{\top} \mathbf{A} & 0
\end{array}\right)
$$

(5) The Casimir functions of the Poisson structure $\mathbf{J}$ depend only on $\boldsymbol{y}$ and are first integrals of the vector field $\boldsymbol{\xi}$.

(6) The symplectic leaves (of dimension 2) of the Poisson structure $\mathbf{J}$ are direct products of the form $\boldsymbol{\gamma} \times \mathbb{R}(\tau)$, where $\boldsymbol{\gamma} \subset \mathbb{R}^{n}(\boldsymbol{y})$ is the trajectory of the vector field $\boldsymbol{\xi}$.

This proposition shows that the Casimir functions and thus the structure of symplectic leaves depend on the properties of solutions of a linear differential equation of the form $\dot{\boldsymbol{y}}=\mathbf{A}^{\top} \boldsymbol{y}$. These properties, in turn, depend on the Jordan canonical form of the matrix $\mathbf{A}$. We are not going to consider the general case in more detail and return to our specific matrix (9).

\subsection{Application to Analysis of the Reduced System (5)}

First of all, note that $\operatorname{Tr} \mathbf{A}=0$, therefore any system that is Hamiltonian with respect to the bracket (8) has a regular invariant measure. This immediately prohibits the existence of attractors and limit cycles both in the entire 5-dimensional phase space and on any nonsingular level manifolds of first integrals.

Note further that by the first statement of Proposition 2, the initial matrix (9) can be replaced by its Jordan form. It is easy to verify that the eigenvalues of this matrix have the form $\pm \sqrt{-1}$ and $\pm \sqrt{-\frac{\left(I_{1}-I_{3}\right)\left(I_{2}-I_{3}\right)}{I_{1} I_{2}}}$. Depending on the sign of the subduplicate, the last two eigenvalues are either purely imaginary or real. These two cases significantly differ from each other in the properties of symplectic leaves and Casimir functions. Let $k^{2}$ denote the absolute value of the subduplicate. Then by a suitable change of 
variables the matrix $\mathbf{A}$ can be reduced to one of the following two block diagonal forms

$$
\mathbf{A}=\left(\begin{array}{cccc}
0 & k & & \\
-k & 0 & & \\
& 0 & 1 \\
& & -1 & 0
\end{array}\right) \quad \text { or } \quad \mathbf{A}=\left(\begin{array}{cccc}
0 & k & & \\
k & 0 & & \\
& 0 & 1 \\
& & -1 & 0
\end{array}\right)
$$

The coordinates in which the matrix $A$ has this form will be denoted, as before, by $y_{1}, y_{2}, y_{3}, y_{4}$.

Let us start with the first case, for which the variables $y$ have the form

$$
\begin{gathered}
y_{1}=I_{1} \omega_{1}-I_{3} \Omega_{1}, y_{2}=I_{2} \omega_{2}-I_{3} \Omega_{2}, \\
y_{3}=\sqrt{I_{1}\left(I_{1}-I_{3}\right)} \omega_{1}, y_{4}=\sqrt{I_{2}\left(I_{2}-I_{3}\right)} \omega_{2} .
\end{gathered}
$$

We begin with a description of the Casimir functions and symplectic leaves. According to statements 5 and 6 of Proposition 2, it is sufficient to analyze the first integrals and integral trajectories of the linear differential equation $\dot{\boldsymbol{y}}=\mathbf{A}^{\top} \boldsymbol{y}$. In this case, the dynamics of this system are quite simple and can be understood as a composition of two uniform rotations in the planes $\left(y_{1}, y_{2}\right)$ and $\left(y_{3}, y_{4}\right)$ with angular velocities 1 and $k$, respectively.

It follows immediately that the Poisson bracket has two "good" quadratic Casimir functions:

$$
C_{1}=y_{1}^{2}+y_{2}^{2} \quad \text { and } \quad C_{2}=y_{3}^{2}+y_{4}^{2} .
$$

(in the second case, the Casimir function $C_{1}$ has the form $y_{1}^{2}-y_{2}^{2}$ ).

Having fixed their values, we obtain a 2-dimensional torus in the space $\mathbb{R}^{4}(\boldsymbol{y})$. If we further introduce two angle variables $\varphi_{1}=\arctan \frac{y_{2}}{y_{1}}$ and $\varphi_{2}=\arctan \frac{y_{4}}{y_{3}}$ and consider $C_{1}, C_{2}, \varphi_{1}, \varphi_{2}$ as a system of (polar) coordinates on $\mathbb{R}^{4}$, then the linear vector field $\boldsymbol{\xi}(\boldsymbol{y})=\mathbf{A}^{\top} \boldsymbol{y}$ defined by the operator $\mathbf{A}$ will have a very simple form:

$$
\boldsymbol{\xi}=(0,0, k, 1)
$$

With an irrational $k$ the trajectory of this vector field is an everywhere dense orbit of a torus and therefore does not admit another global integral (which, as mentioned above, would be a Casimir function of the bracket under consideration). However, a local integral exists and can be written as

$$
C_{3}=\varphi_{1}-k \varphi_{2} .
$$

Remark 1. It is useful to note that the irrational orbit is the same for all tori of the form $\left\{C_{1}=\right.$ const $_{1}, C_{2}=$ const $\left._{2}\right\}$. In other words, the number $k$ in the third integral $C_{3}$ does not depend on the values of the integrals $C_{1}$ and $C_{2}$, but is a constant defined by the Poisson tensor. Its invariant meaning is that $k$ is the ratio of eigenvalues of the matrix $\mathbf{A}$ of the form (9) (or the canonical form (11)). For more general matrices $\mathbf{A}$, the situation is similar: the ratios of eigenvalues play an important role and are included as parameters in the explicit formulae for integrals.

The information provided above is quite sufficient to integrate by quadratures any Hamiltonian system on $\mathfrak{g}^{*}$.

Proposition 3. Consider an arbitrary Hamiltonian system with respect to the Poisson structure (10) that corresponds to the matrix $\mathbf{A}$ of the form (11) (the first case). Then the system is integrable by quadratures.

Proof. There is no doubt about the possibility of integrating the system by quadratures, because we know the explicit formulae for Casimir functions and, therefore, can restrict the system to a symplectic leaf, after which it can be integrated as a Hamiltonian system with one degree of freedom.

It only remains to explain exactly how it should be done in this case.

First of all, note that the Poisson bracket has two "good" quadratic Casimir functions:

$$
C_{1}=y_{1}^{2}+y_{2}^{2} \quad \text { and } \quad C_{2}=y_{3}^{2}+y_{4}^{2} .
$$

(In the second case, the Casimir function $C_{1}$ has the form $y_{1}^{2}-y_{2}^{2}$ ). 
If we fix their values, we get a 2-dimensional torus in the space $\mathbb{R}^{4}(\boldsymbol{y})$. If we further introduce two angle variables $\varphi_{1}=\arctan \frac{y_{2}}{y_{1}}$ and $\varphi_{2}=\arctan \frac{y_{4}}{y_{3}}$ and consider $C_{1}, C_{2}, \varphi_{1}, \varphi_{2}$ as a system of (polar) coordinates on $\mathbb{R}^{4}$, then the linear vector field defined by the operator $\mathbf{A}$ will have a very simple form:

$$
\boldsymbol{\xi}=(0,0, k, 1) \text {. }
$$

With an irrational $k$ the trajectory of this vector field is an everywhere dense orbit of a torus and therefore does not admit another global integral (which, as mentioned above, would be a Casimir function of the bracket under consideration). However, a local integral exists and can be written as

$$
C_{3}=\varphi_{1}-k \varphi_{2} .
$$

Consider an arbitrary Hamiltonian $H$ on $\mathbb{R}^{5}$. In terms of the variables $C_{1}, C_{2}, \varphi_{1}, \varphi_{2}, \tau$, the corresponding Hamiltonian system can be written as

$$
\dot{C}_{1}=0, \quad \dot{C}_{2}=0, \quad \dot{\varphi}_{1}=k F, \quad \dot{\varphi}_{2}=F, \quad \dot{\tau}=G,
$$

where $F$ and $G$ are certain functions. Now if we rescale time as $d t=F d t^{\prime}$, the equations take the form

$$
\dot{C}_{1}=0, \quad \dot{C}_{2}=0, \quad \dot{\varphi}_{1}=k, \quad \dot{\varphi}_{2}=1, \quad \dot{\tau}=\tilde{G},
$$

where $\tilde{G}=\frac{G}{F}$ is a certain function of all the variables. Finally, note that the Hamiltonian of the system $H$ is a first integral, and this allows us to express the variable $\tau$ in terms of $H, C_{1}, C_{2}, \varphi_{1}, \varphi_{2}$. Thus, the equations of motion take the form

$$
\dot{\varphi}_{1}=1, \quad \dot{\varphi}_{2}=k, \quad \dot{\tau}=\tilde{G}\left(\varphi_{1}, \varphi_{2} ; C_{1}, C_{2}, H\right),
$$

and are immediately integrated and $\tau\left(t^{\prime}\right)=\int \tilde{G}\left(t^{\prime}, k t^{\prime} ; C_{1}, C_{2}, H\right) d t^{\prime}$.

Remark 2. For the Hamiltonian (7) considered in the following sections

$$
\begin{gathered}
F=\tau, \\
G=\frac{I_{\mathrm{s}} \sqrt{c_{2}}}{I_{3}\left(I_{\mathrm{S}}+I_{3}\right)}\left(\sqrt{\frac{c_{1}}{I_{1}\left(I_{1}-I_{3}\right)}} \sin \varphi_{1} \cos \varphi_{2}-\sqrt{\frac{c_{1}}{I_{2}\left(I_{2}-I_{3}\right)}} \cos \varphi_{1} \sin \varphi_{2}\right)+c_{1} \frac{\left(I_{1}-I_{2}\right)}{2 k \operatorname{det} \mathbf{I}} \sin \left(2 \varphi_{1}\right) .
\end{gathered}
$$

In fact, we have used the two-dimensionality of symplectic leaves for this explicit integration. In the standard situation where symplectic leaves are closed submanifolds explicitly defined by the Casimir functions, two-dimensionality means that we integrate a Hamiltonian system with one degree of freedom. Here we have also implicitly used the existence of a local integral $C_{3}$.

The nonexistence of a global Casimir function, or rather the fact that a symplectic leaf is not a "properly embedded submanifold" but a direct product of the irrational torus orbit by $\mathbb{R}$, leads to a significant difference of the global dynamics of the system from what we are used to seeing in systems with one degree of freedom. One of the "generally accepted" properties of such systems is the following. If the Hamiltonian of a system is positive definite (or, more generally, its level sets $\{H=$ const $\}$ are compact), then in the case of one degree of freedom almost all trajectories of the system are closed. It is not so in this case. The open region of the phase space turns out to be filled with nonclosed trajectories such as irrational orbits. A detailed topological analysis of the system is provided in the following subsections.

\section{Qualitative Analysis of the Reduced System}

\section{1. $\quad$ Fixed Points}

First of all, let us consider the equilibrium points of system (5) and analyze their stability. In terms of the original variables $\boldsymbol{z}=\left(\Omega_{1}, \Omega_{2}, \omega_{1}, \omega_{2}, \omega_{3}\right)$, the set of fixed points is defined as

$$
\mathcal{M}_{*}^{3}=\left\{\omega_{3}=0, I_{\mathrm{S}}\left(\Omega_{1} \omega_{2}-\Omega_{2} \omega_{1}\right)+\left(I_{1}-I_{2}\right) \omega_{1} \omega_{2}=0\right\} .
$$

For a clear and transparent presentation of results it is more convenient to analyze the fixed points of the system not in the entire phase space but on individual level surfaces where the solution is represented by quadratures (see the previous section):

$$
\mathcal{M}_{c, h}=\left\{C_{1}(\boldsymbol{z})=c_{1}, C_{2}(\boldsymbol{z})=c_{2}, H(\boldsymbol{z})=h\right\},
$$


here $H, C_{1}, C_{2}$ are defined by the relations (6) and (7). The equilibrium points on the manifold $\mathcal{M}_{c, h}$ are isolated. There are two cases that need to be considered separately. They differ in the properties of the function $C_{1}$ :

(1) $\left(I_{1}-I_{3}\right)\left(I_{2}-I_{3}\right)>0$ the Casimir function $C_{1}$ is a sign definite function;

(2) $\left(I_{1}-I_{3}\right)\left(I_{2}-I_{3}\right)<0$ the Casimir function is either positive or negative definite; in this case it is indefinite.

We emphasize that such a significant difference between these cases is due to the fact that the corresponding Poisson brackets are essentially nonisomorphic. That is, the difference in the dynamic behavior of the system in this case is due to the bracket (or, which is the same, a corresponding 5-dimensional Lie algebra).

In our further analysis of the system we will assume throughout (without loss of generality) that the axes $C x_{1}$ and $C x_{2}$ are chosen so that the inequality $I_{2}<I_{1}$ holds. As can be easily seen, the function $C_{1}$ is sign indefinite if the value of $I_{3}$ satisfies the inequality $I_{2}<I_{3}<I_{1}$.

$\left(\boldsymbol{I}_{1}-\boldsymbol{I}_{\mathbf{3}}\right)\left(\boldsymbol{I}_{\mathbf{2}}-\boldsymbol{I}_{\mathbf{3}}\right)>\mathbf{0}$. The transformation to polar coordinates on $\mathcal{M}_{c, h}$ from the previous section is as follows:

$$
\begin{gathered}
\omega_{1}=\sqrt{\frac{c_{1}}{I_{1}\left(I_{1}-I_{3}\right)}} \sin \varphi_{1}, \quad \omega_{2}=\sqrt{\frac{c_{1}}{I_{2}\left(I_{2}-I_{3}\right)}} \cos \varphi_{1}, \\
\Omega_{1}=\sqrt{\frac{I_{1} c_{1}}{I_{1}-I_{3}}} \frac{\sin \varphi_{1}}{I_{3}}-\sqrt{c_{2}} \frac{\sin \varphi_{2}}{I_{3}}, \Omega_{2}=\sqrt{\frac{I_{2} c_{1}}{I_{2}-I_{3}} \frac{\cos \varphi_{1}}{I_{3}}-\sqrt{c_{2}} \frac{\cos \varphi_{2}}{I_{3}},}
\end{gathered}
$$

where $\varphi_{1}, \varphi_{2} \in[0,2 \pi)$. In this case, the fixed points on $\mathcal{M}_{c, h}$ are defined by the following relations:

$$
\begin{gathered}
G=0, \quad Q=h I_{3}^{2}\left(I_{3}+I_{\mathrm{s}}\right) \\
G=\frac{I_{\mathrm{s}} \sqrt{c_{2}}}{I_{3}\left(I_{\mathrm{s}}+I_{3}\right)}\left(\sqrt{\frac{c_{1}}{I_{1}\left(I_{1}-I_{3}\right)}} \sin \varphi_{1} \cos \varphi_{2}-\sqrt{\frac{c_{1}}{I_{2}\left(I_{2}-I_{3}\right)}} \cos \varphi_{1} \sin \varphi_{2}\right)+c_{1} \frac{\left(I_{1}-I_{2}\right)}{2 k \operatorname{det} \mathbf{I}} \sin \left(2 \varphi_{1}\right), \\
Q=\frac{I_{\mathrm{s}} c_{2}}{2}+\frac{c_{1}}{2}\left(\frac{I_{\mathrm{s}} I_{1}+I_{3}^{2}}{I_{1}-I_{3}} \sin ^{2} \varphi_{1}+\frac{I_{\mathrm{s}} I_{2}+I_{3}^{2}}{I_{2}-I_{3}} \cos ^{2} \varphi_{1}\right)- \\
-I_{\mathrm{s}} \sqrt{c_{2}}\left(\sqrt{\frac{I_{1} c_{1}}{I_{1}-I_{3}}} \sin \varphi_{1} \sin \varphi_{2}+\sqrt{\frac{I_{2} c_{1}}{I_{2}-I_{3}}} \cos \varphi_{1} \cos \varphi_{2}\right) .
\end{gathered}
$$

The first of these equations can be conveniently rewritten as

$$
g\left(\varphi_{1}, \varphi_{2}\right)=\frac{\frac{2 I_{\mathrm{S}}}{\left(I_{\mathrm{S}}+I_{3}\right)} \sqrt{\frac{c_{2}}{c_{1}}}-g\left(\varphi_{1}, \varphi_{2}\right)=0,}{\sqrt{I_{1}\left(I_{1}-I_{3}\right)} \sin \varphi_{2} \cos \varphi_{1}-\sqrt{I_{2}\left(I_{2}-I_{3}\right)} \sin \varphi_{1} \cos \varphi_{2}} .
$$

Hence, taking into account that the function $H(\boldsymbol{z})$ is positive definite, we get the following statement:

if the coordinates of the point $\left(\varphi_{1}, \varphi_{2}\right)$ satisfy the inequality $g\left(\varphi_{1}, \varphi_{2}\right)>0$, then there are positive values of $c_{1}, c_{2}$, $h$ that satisfy equations (18), i.e., on a given $\mathcal{M}_{c, h}$ the point $\left(\varphi_{1}, \varphi_{2}\right)$ defines the equilibrium point of system (5).

Remark 1. In many cases, the question of the number and stability of fixed points on a given $\mathcal{M}_{c, h}$ (i.e., for fixed values of the first integrals $\left.c_{1}, c_{2}, h\right)$ is more essential. We are not going to discuss this issue here. We only note that in our case, using the well-known formula

$$
\sum_{\text {crit. points }} \text { ind }=\chi\left(\mathcal{M}_{c, h}^{2}\right),
$$

where $\chi$ is the Euler characteristic of the two-dimensional surface, we obtain

$$
\sum_{\text {st }}-\sum_{\text {un }}=2-2 g,
$$


where $\sum_{\text {st }}, \sum_{\text {un }}$ are the sums of stable and unstable fixed points, and $g$ is the number of handles of surface $M_{c, h}$ (i.e., its genus).

To investigate the stability of fixed points, we use the Poisson bracket (8). According to the general criterion obtained in the Appendix, the stability of the fixed point $z_{0}$ is defined by the sign of the function

$$
\mu_{h}\left(\boldsymbol{z}_{0}\right)=\operatorname{det}\left\|\begin{array}{ll}
\boldsymbol{\xi}(\boldsymbol{\xi}(H)) & \boldsymbol{\xi}(\boldsymbol{\eta}(H)) \\
\boldsymbol{\xi}(\boldsymbol{\eta}(H)) & \boldsymbol{\eta}(\boldsymbol{\eta}(H))
\end{array}\right\|,
$$

where $\boldsymbol{\xi}, \boldsymbol{\eta}$ are the vector fields (in the neighborhood of fixed point $\boldsymbol{z}_{\mathbf{0}}$ ) locally defined by the decomposition of the Poisson tensor $\mathbf{J}=\boldsymbol{\xi} \wedge \boldsymbol{\eta}$.

In this case, as shown in the previous section, this decomposition exists globally, and the fields $\boldsymbol{\xi}, \boldsymbol{\eta}$ in the variables $\boldsymbol{z}=\left(\Omega_{1}, \Omega_{2}, \omega_{1}, \omega_{2}, \omega_{3}\right)$ become

$$
\boldsymbol{\xi}=\left(\Omega_{2}-\omega_{2}, \omega_{1}-\Omega_{1}, \frac{I_{2}-I_{3}}{I_{1}} \omega_{2}, \frac{I_{3}-I_{1}}{I_{2}} \omega_{1}, 0\right), \quad \boldsymbol{\eta}=(0,0,0,0,1) .
$$

Calculating the value of (19) and restricting it to the manifold $\mathcal{M}_{c, h}$, we obtain

$$
\begin{aligned}
\widetilde{\mu}_{h}\left(\varphi_{1}, \varphi_{2}\right)=\frac{\operatorname{det} \mathbf{I}}{I_{\mathrm{s}} \sqrt{c_{1} c_{2}}} \mu_{h}\left(\varphi_{1}, \varphi_{2}\right) & =\frac{\sqrt{I_{1}}\left(I_{2} \sin ^{2} \varphi_{1}-\left(I_{1}-I_{3}\right) \cos ^{2} \varphi_{1}\right)}{\sqrt{I_{1}-I_{3}}} \frac{\sin \varphi_{2}}{\sin \varphi_{1}}+ \\
& +\frac{\sqrt{I_{2}}\left(I_{1} \cos ^{2} \varphi_{1}-\left(I_{2}-I_{3}\right) \sin ^{2} \varphi_{1}\right)}{\sqrt{I_{2}-I_{3}}} \frac{\cos \varphi_{2}}{\cos \varphi_{1}}
\end{aligned}
$$

Theorem 1. Let $\varphi^{*}=\left(\varphi_{1}^{*}, \varphi_{2}^{*}\right)$ be a fixed point of system (5) on the integral surface $\mathcal{M}_{c, h}$. Then

$\varphi^{*}$ is stable (in the sense of Lyapunov) if $\widetilde{\mu}_{h}\left(\varphi_{1}^{*}, \varphi_{2}^{*}\right)>0$,

$\varphi^{*}$ is unstable if $\widetilde{\mu}_{h}\left(\varphi_{1}^{*}, \varphi_{2}^{*}\right)<0$.

The issue of stability of fixed points $\varphi^{*}$ in which $\widetilde{\mu}_{h}\left(\varphi_{1}^{*}, \varphi_{2}^{*}\right)=0$ requires further investigation.

Remark 2. Here and in the sequel, stability is considered in relation to the perturbation of variables $\left(\Omega_{1}, \Omega_{2}, \omega_{1}, \omega_{2}, \omega_{3}\right)$.

For fixed moments of inertia, the regions of stable and unstable fixed points are shown in Fig. 3.

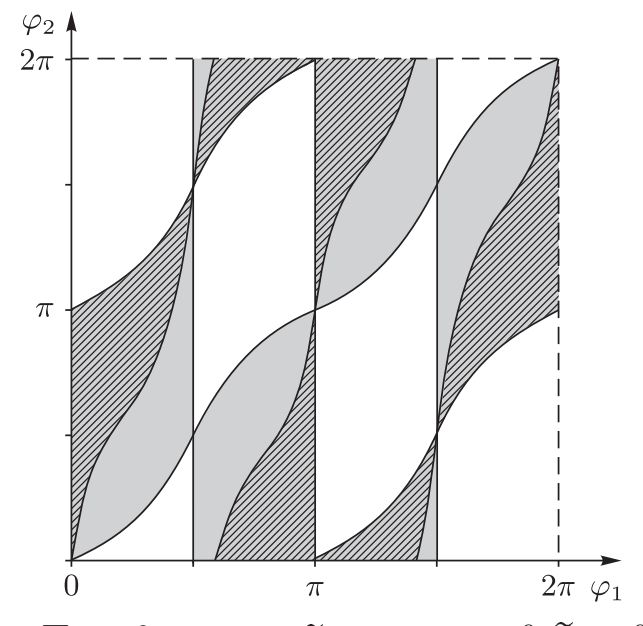

$\square g<0 ; \square g>0, \widetilde{\mu}_{h}>0 ; \mathbb{Q} g>0, \widetilde{\mu}_{h}<0 ;$

Fig. 3. Regions of stable (grey) and unstable (hatched) equilibria $\left(I_{1}=5.2, I_{2}=4.3, I_{3}=3.9, I_{\mathrm{S}}=3.6\right)$.

If the inner body is dynamically symmetrical $I_{1}=I_{2} \neq I_{3}$, the first of Eqs. (18) greatly simplifies to give

$$
\sin \varphi_{1} \cos \varphi_{2}-\cos \varphi_{1} \sin \varphi_{2}=0 .
$$


Hence we have two solutions:

$$
\varphi_{2}=\varphi_{1}, \quad \varphi_{2}=\varphi_{1}+\pi
$$

The first solution is always stable $\left(\widetilde{\mu}_{h}>0\right)$, while the second is always unstable $\left(\widetilde{\mu}_{h}<0\right)$.

$\left(\boldsymbol{I}_{1}-\boldsymbol{I}_{3}\right)\left(\boldsymbol{I}_{2}-\boldsymbol{I}_{3}\right)<\mathbf{0}$. In this case, the transformation to local coordinates has the following form:

$$
\begin{aligned}
& \text { if } c_{1}>0, \text { then } \omega_{1}= \pm \sqrt{\frac{c_{1}}{I_{1}\left(I_{1}-I_{3}\right)}} \cosh \varphi_{1}^{ \pm}, \quad \omega_{2}=-\sqrt{\frac{c_{1}}{I_{2}\left(I_{3}-I_{2}\right)}} \sinh \varphi_{1}^{ \pm}, \\
& \Omega_{1}= \pm \sqrt{\frac{I_{1} c_{1}}{I_{1}-I_{3}}} \frac{\cosh \varphi_{1}^{ \pm}}{I_{3}}-\frac{\sqrt{c_{2}}}{I_{3}} \sin \varphi_{2}, \Omega_{2}=-\sqrt{\frac{I_{2} c_{1}}{I_{3}-I_{2}}} \frac{\sinh \varphi_{1}^{ \pm}}{I_{3}}-\frac{\sqrt{c_{2}}}{I_{3}} \cos \varphi_{2}, \\
& \text { if } c_{1}<0, \text { then } \omega_{1}=-\sqrt{\frac{c_{1}}{I_{1}\left(I_{3}-I_{1}\right)}} \sinh \varphi_{1}^{ \pm}, \quad \omega_{2}= \pm \sqrt{\frac{c_{1}}{I_{2}\left(I_{2}-I_{3}\right)}} \cosh \varphi_{1}^{ \pm}, \\
& \Omega_{1}=-\sqrt{\frac{I_{1} c_{1}}{I_{3}-I_{1}}} \frac{\sinh \varphi_{1}^{ \pm}}{I_{3}}-\sqrt{c_{2}} \frac{\sin \varphi_{2}}{I_{3}}, \Omega_{2}= \pm \sqrt{\frac{I_{2} c_{1}}{I_{2}-I_{3}}} \frac{\cosh \varphi_{1}^{ \pm}}{I_{3}}-\sqrt{c_{2}} \frac{\cos \varphi_{2}}{I_{3}},
\end{aligned}
$$

the sign \pm arises from the fact that in each of these two cases, the equation $C_{1}=c_{1}$ defines a pair of hyperbolas, one of which is parameterized by the variable $\varphi_{1}^{+}$, and the other by $\varphi_{1}^{-}$.

As we can see, for stability analysis of the fixed points in this case it is sufficient to substitute in the above formulas:

for $c_{1}<0: \varphi_{1}^{ \pm}=i \varphi_{1}$ where $\varphi_{1} \in(-\infty, \infty)$;

for $c_{1}>0: \varphi_{1}^{ \pm}=\frac{\pi}{2}-i \varphi_{1}$ where $\varphi_{1} \in(-\infty, \infty)$.

For fixed parameters, the regions of stable and unstable fixed points are shown in Fig. 4.

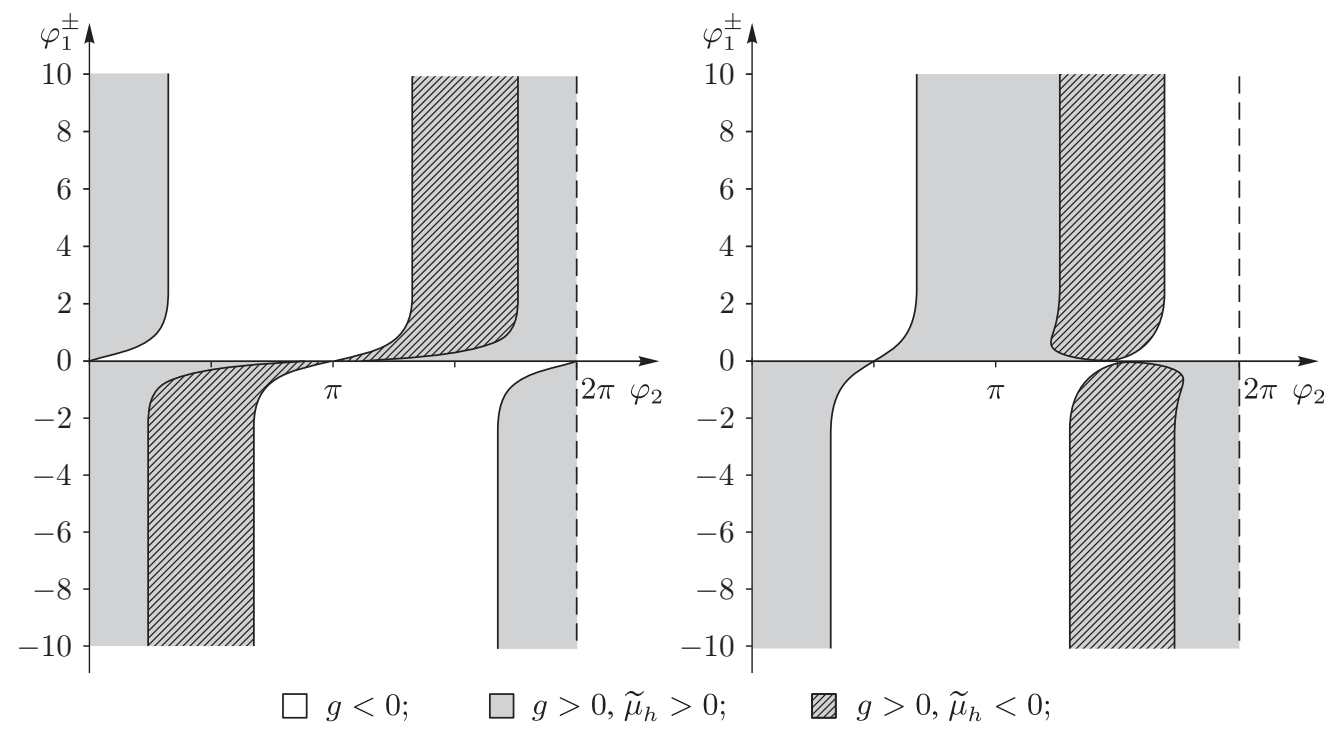

Fig. 4. Regions of stable (grey) and unstable (hatched) equilibria, left $c_{1}<0$, and right $c_{1}>0\left(I_{1}=3.3, I_{2}=5.2, I_{3}=4\right.$, $\left.I_{\mathrm{S}}=3.6\right)$. On each of the planes $\left(\varphi_{1}^{+}, \varphi_{2}\right)$ and $\left(\varphi_{1}^{-}, \varphi_{2}\right)$ correspond to different hyperbolas, and these regions are identical.

\subsection{Topological Analysis of Integral Manifolds and Classification of Trajectories}

In the previous sections, a solution in terms of quadratures (14) and (15) was found on the level surface $\mathcal{M}_{c, h}$ and a set of fixed points was studied. In this section, we investigate the structure of the manifold $\mathcal{M}_{c, h}$ itself and study the behavior of trajectories on it using the methods of topological analysis. 
We first recall some definitions and notation, following mainly [Bolsinov et al., 2010]. We denote by $\Phi=\left(C_{1}, C_{2}, H\right): \mathcal{M}^{5} \rightarrow \mathbb{R}^{3}$ the integral map of the system (5):

$$
\boldsymbol{z} \mapsto \Phi(\boldsymbol{z})=\left(C_{1}(\boldsymbol{z}), C_{2}(\boldsymbol{z}), H(\boldsymbol{z})\right) \in \mathbb{R}^{3} .
$$

The region of possible motions (RPM) $\Phi\left(\mathcal{M}^{5}\right)$ is the full image of the phase space in the space of first integrals. To each point $\left(c_{1}, c_{2}, h\right) \in \Phi\left(\mathcal{M}^{5}\right)$ there corresponds the integral manifold $\mathcal{M}_{c, h}$ of the system, which, generally speaking, can contain several connected components.

The set of critical points of the integral map in this case is defined as

$$
S=\left\{\boldsymbol{z} \in \mathcal{M}^{5} \mid \operatorname{rank} \mathrm{d} \Phi(\boldsymbol{z})<3\right\},
$$

The image of the corresponding set in the space of first integrals will be denoted likewise:

$$
\Sigma=\Phi(S) \text {. }
$$

We will call the region of possible motions $\Phi\left(\mathcal{M}^{5}\right)$ depicted in the space of first integrals together with the critical set image $\Sigma$ a bifurcation diagram.

The critical set. Using undetermined Lagrange multipliers, the set of critical points is defined as a solution of the system of equations

$$
\lambda_{1} \frac{\partial C_{1}}{\partial \boldsymbol{z}}+\lambda_{2} \frac{\partial C_{2}}{\partial \boldsymbol{z}}+2 \lambda_{3}\left(I_{3}+I_{\mathrm{s}}\right) \frac{\partial H}{\partial \boldsymbol{z}}=0,
$$

where $\boldsymbol{z}$ and the relations of the undetermined multipliers $\lambda_{1}, \lambda_{2}, \lambda_{3}$ are unknown. The system (21) can be expressed in matrix form

$$
\left(\begin{array}{ccccc}
\lambda_{2} I_{3}^{2}+\lambda_{3} I_{\mathrm{s}} & -\lambda_{2} I_{1} I_{3} & 0 & 0 & 0 \\
-\lambda_{2} I_{1} I_{3} & \left(\lambda_{1}+\lambda_{2}\right) I_{1}^{2}+\lambda_{3} I_{1}-\lambda_{1} I_{3} & 0 & 0 & 0 \\
0 & 0 & \lambda_{2} I_{3}^{2}+\lambda_{3} I_{\mathrm{s}} & -\lambda_{2} I_{2} I_{3} & 0 \\
0 & 0 & -\lambda_{2} I_{2} I_{3} & \left(\lambda_{1}+\lambda_{2}\right) I_{2}^{2}+\lambda_{3} I_{2}-\lambda_{2} I_{3} & 0 \\
0 & 0 & 0 & 0 & \lambda_{3}\left(I_{3}+I_{\mathrm{s}}\right)
\end{array}\right)\left(\begin{array}{c}
\Omega_{1} \\
\omega_{1} \\
\Omega_{2} \\
\omega_{2} \\
\omega_{3}
\end{array}\right)=0
$$

As is well known, the condition for the existence of a nontrivial solution of such a system is the vanishing of the determinant of the corresponding matrix of coefficients, which is expressed as

$$
\begin{gathered}
\Lambda_{1} \Lambda_{2} \lambda_{3}=0, \\
\Lambda_{i}=\left(\lambda_{1}\left(I_{i}-I_{3}\right)+\lambda_{3}\right)\left(\lambda_{2} I_{3}^{2}+\lambda_{3} I_{\mathrm{s}}\right)+\lambda_{2} \lambda_{3} I_{i} I_{\mathrm{s}}, \quad i=1,2 .
\end{gathered}
$$

Depending on the number of multipliers vanishing in this expression, we obtain the following possible solutions of the original system

1. The case $\lambda_{1}=\lambda_{3}=0, \lambda_{2} \neq 0$ (in this case, all three multipliers in Eq. (23) vanish). The solutions of the system (22) form a particular three-parameter family that is defined by

$$
S_{1}=\left\{\Omega_{1}=\frac{I_{1}}{I_{3}} \omega_{1}, \quad \Omega_{2}=\frac{I_{2}}{I_{3}} \omega_{2}\right\} .
$$

In this case, $C_{2}=0$ and $d C_{2}=0$.

2. The case $\lambda_{2}=\lambda_{3}, \lambda_{1} \neq 0$ (all three multipliers in Eq. (23) vanish also). The three-parameter family of solutions is defined by

$$
S_{2}=\left\{\omega_{1}=0, \quad \omega_{2}=0\right\}
$$

In this case, $C_{1}=0$ and $d C_{1}=0$.

3. The case $\Lambda_{1}=\Lambda_{2}=0, \lambda_{3} \neq 0$. In this case,

$$
\frac{\lambda_{3}}{\lambda_{1}}=I_{3}, \quad \frac{\lambda_{3}}{\lambda_{2}}=-I_{3}\left(1+\frac{I_{3}}{I_{\mathrm{s}}}\right),
$$

and the solutions of the system (22) form a two-parameter family defined by

$$
S_{3}=\left\{\Omega_{1}=-\frac{I_{1}}{I_{\mathrm{s}}} \omega_{1}, \quad \Omega_{2}=-\frac{I_{2}}{I_{\mathrm{s}}} \omega_{2}, \quad \omega_{3}=0\right\} .
$$


4. The case $\Lambda_{1}=0, \Lambda_{2} \lambda_{3} \neq 0$. In this case, the undefined multipliers satisfy the relation

$$
\frac{\lambda_{3}}{\lambda_{1}}=-\frac{\left(I_{1}-I_{3}\right)\left(I_{3}^{2}+I_{\mathrm{s}} \frac{\lambda_{3}}{\lambda_{2}}\right)}{I_{1} I_{\mathrm{s}}+I_{3}^{2}+I_{\mathrm{s}} \frac{\lambda_{3}}{\lambda_{2}}}
$$

and the two-parameter family of solutions $(22)$ is written as

$$
S_{4}=\left\{\Omega_{1}=\frac{I_{1} I_{3}}{I_{3}^{2}+I_{\mathrm{s}} \frac{\lambda_{3}}{\lambda_{2}}} \omega_{1}, \quad \Omega_{2}=0, \quad \omega_{2}=0, \quad \omega_{3}=0\right\},
$$

where one of the parameters is the relation $\frac{\lambda_{3}}{\lambda_{2}}$.

5. The case $\Lambda_{2}=0, \Lambda_{1} \lambda_{3} \neq 0$. In this case,

$$
\frac{\lambda_{3}}{\lambda_{1}}=-\frac{\left(I_{2}-I_{3}\right)\left(I_{3}^{2}+I_{\mathrm{s}} \frac{\lambda_{3}}{\lambda_{2}}\right)}{I_{2} I_{3}+I_{3}^{2}+I_{\mathrm{s}} \frac{\lambda_{3}}{\lambda_{2}}}
$$

and the two-parameter family of solutions (22) is expressed as

$$
S_{5}=\left\{\Omega_{1}=0, \quad \Omega_{2}=\frac{I_{2} I_{3}}{I_{3}^{2}+I_{\mathrm{s}} \frac{\lambda_{3}}{\lambda_{2}}} \omega_{2}, \quad \Omega_{2}=0, \quad \omega_{1}=0, \quad \omega_{3}=0\right\},
$$

where $\frac{\lambda_{3}}{\lambda_{2}}$ is also one of the parameters.

6. The case $\lambda_{3}=0, \Lambda_{1} \Lambda_{2} \neq 0$. Hence, in particular, it follows that $\lambda_{1} \lambda_{2} \neq 0$, and the solutions of the system (22) form a one-parameter family defined by

$$
S_{6}=\left\{\Omega_{1}=0, \quad \Omega_{2}=0, \quad \omega_{1}=0, \quad \omega_{2}=0\right\} .
$$

Remark 3. In the first five cases rank $\mathrm{d} \Phi=2$, while in the last case $\operatorname{rank} \mathrm{d} \Phi=1$.

Before describing the possible bifurcation diagrams of the system, we assume throughout (without loss of generality) that we have fixed $I_{2}<I_{1}$, and the cases $I_{3}<I_{2}<I_{1}$ and $I_{2}<I_{1}<I_{3}$ reduce to each other by replacing the function $C_{1}(\boldsymbol{z})$ with $-C_{1}(\boldsymbol{z})$. Therefore, in what follows we restrict ourselves to a detailed analysis of the two qualitatively different cases

$$
I_{3}<I_{2}<I_{1} \quad \text { and } \quad I_{2}<I_{3}<I_{1} .
$$

The bifurcation diagram and integral surfaces for the case $I_{3}<I_{2}<I_{1}$. Let us now consider the images of all the previously found families of critical points $S_{i}, i=1 \ldots 6$ in the space of values of the integrals $\mathbb{R}^{3}=\left\{\left(c_{1}, c_{2}, h\right)\right\}$.

Using the parametric representation given above, it can be shown that the image of the first family $S_{1}$ is a part of the coordinate plane $c_{2}=0$ satisfying the following conditions

$$
\Sigma_{1}=\left\{\left(c_{1}, 0, h\right) \mid c_{1} \geqslant 0, \quad h \geqslant \frac{I_{1} I_{\mathrm{s}}+I_{3}^{2}}{2 I_{3}^{2}\left(I_{1}-I_{3}\right)\left(I_{3}+I_{\mathrm{s}}\right)} c_{1}\right\} .
$$

Similarly, for the family $S_{2}$ we get a part of the coordinate plane $c_{1}=0$ :

$$
\Sigma_{2}=\left\{\left(0, c_{2}, h\right) \mid c_{2} \geqslant 0, \quad h \geqslant \frac{I_{\mathrm{s}}}{2 I_{3}^{2}\left(I_{3}+I_{\mathrm{s}}\right)} c_{2}\right\} .
$$

The image of the family $S_{3}$ is a part of the plane $h=\frac{I_{\mathrm{s}} c_{2}-\left(I_{3}+I_{\mathrm{s}}\right) c_{1}}{2 I_{3}\left(I_{3}+I_{\mathrm{s}}\right)^{2}}$, which can naturally be expressed in parametric form

$$
\begin{gathered}
\Sigma_{3}=\left\{\left(c_{1}, c_{2}, h\right) \mid c_{1}=I_{1}\left(I_{1}-I_{3}\right) u+I_{2}\left(I_{2}-I_{3}\right) v, \quad c_{2}=\frac{\left(I_{3}+I_{\mathrm{S}}\right)^{2}}{I_{\mathrm{s}}^{2}}\left(I_{1}^{2} u+I_{2}^{2} v\right),\right. \\
\left.h=\frac{I_{1}\left(I_{1}+I_{\mathrm{s}}\right)}{2 I_{\mathrm{s}}\left(I_{3}+I_{\mathrm{s}}\right)} u+\frac{I_{2}\left(I_{2}+I_{\mathrm{S}}\right)}{2 I_{\mathrm{S}}\left(I_{3}+I_{\mathrm{s}}\right)} v, u \geqslant 0, v \geqslant 0\right\} .
\end{gathered}
$$


The families $S_{4}$ and $S_{5}$ in the space of integrals correspond to two conical surfaces

$$
\begin{aligned}
& \Sigma_{4}=\left\{\left(c_{1}, c_{2}, h\right) \mid\left(\frac{2 I_{3}^{2}\left(I_{3}+I_{\mathrm{s}}\right)}{I_{\mathrm{s}}} h-\frac{\left(I_{1} I_{\mathrm{S}}+I_{3}^{2}\right)}{I_{\mathrm{s}}\left(I_{1}-I_{3}\right)} c_{1}-c_{2}\right)^{2}=\frac{4 I_{1}}{I_{1}-I_{3}} c_{1} c_{2}, \quad c_{1}, c_{2}, h>0\right\}, \\
& \Sigma_{5}=\left\{\left(c_{1}, c_{2}, h\right) \mid\left(\frac{2 I_{3}^{2}\left(I_{3}+I_{\mathrm{s}}\right)}{I_{\mathrm{s}}} h-\frac{\left(I_{2} I_{\mathrm{S}}+I_{3}^{2}\right)}{I_{\mathrm{s}}\left(I_{2}-I_{3}\right)} c_{1}-c_{2}\right)^{2}=\frac{4 I_{2}}{I_{2}-I_{3}} c_{1} c_{2}, \quad c_{1}, c_{2}, h>0\right\} .
\end{aligned}
$$

As can be seen from these relations, both surfaces are tangent to the coordinate planes $\left\{c_{1}=0\right\}$ and $\left\{c_{2}=0\right\}$. Note that they are tangent to the plane $c_{1}=0$ along a common straight line. The typical form of the bifurcation surfaces $\Sigma_{3}, \Sigma_{4}, \Sigma_{5}$ in this case is shown in Fig. 5 . The family $S_{6}$ corresponds to the vertical ray

$$
\Sigma_{6}=\left\{\left(c_{1}, c_{2}, h\right) \mid c_{1}=c_{2}=0, h \geqslant 0\right\} .
$$

Since the sets $\Sigma_{i}, i=1, \ldots, 6$ are invariant under the dilation $c_{1} \rightarrow \lambda c_{1}, c_{2} \rightarrow \lambda c_{2}, h \rightarrow \lambda h$, all sections of the bifurcation diagram formed by the intersection of the diagram with the plane $h=$ const (except for $h=0$ ) are similar to each other (the similarity is due to the fact that the trajectories (5) are invariant under the change of variables $\left.\boldsymbol{z} \rightarrow \sqrt{\lambda} \boldsymbol{z}, \boldsymbol{z}=\left(\Omega_{1}, \Omega_{2}, \omega_{1}, \omega_{2}, \omega_{3}\right)\right)$. Therefore, instead of the threedimensional diagram we will consider its sections formed by the intersection with the plane $h=$ const. The corresponding section is shown in Fig. 6.

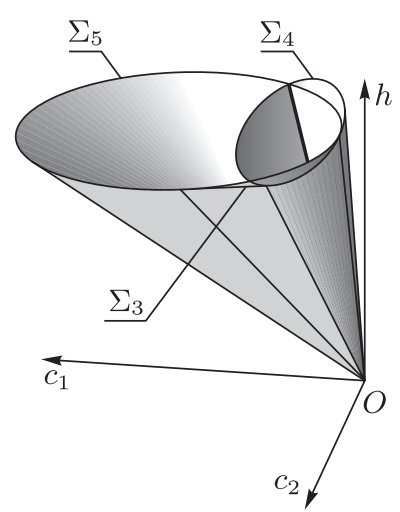

Fig. 5. The typical form of the bifurcation surfaces $\Sigma_{3}, \Sigma_{4}, \Sigma_{5}$ for the case $I_{3}<I_{2}<I_{1}\left(I_{1}=5.2, I_{2}=4.3, I_{3}=3.9\right.$, $\left.I_{\mathrm{S}}=3.6\right)$.

In order to determine the type of the integral surface $\mathcal{M}_{c, h}$ for each of the regions in the bifurcation diagram, we will construct their projections onto the torus defined by the angle variables $\left(\varphi_{1}, \varphi_{2}\right) \bmod 2 \pi$, see Fig. 6. Since

$$
\begin{gathered}
\omega_{3}= \pm \sqrt{2 h-\frac{2 Q}{I_{3}^{2}\left(I_{3}+I_{\mathrm{s}}\right)}}, \\
Q=\frac{I_{\mathrm{s}} c_{2}}{2}+\frac{c_{1}}{2}\left(\frac{I_{\mathrm{s}} I_{1}+I_{3}^{2}}{I_{1}-I_{3}} \sin ^{2} \varphi_{1}+\frac{I_{\mathrm{s}} I_{2}+I_{3}^{2}}{I_{2}-I_{3}} \cos ^{2} \varphi_{1}\right)- \\
-I_{\mathrm{s}} \sqrt{c_{2}}\left(\sqrt{\frac{I_{1} c_{1}}{I_{1}-I_{3}}} \sin \varphi_{1} \sin \varphi_{2}+\sqrt{\frac{I_{2} c_{1}}{I_{2}-I_{3}}} \cos \varphi_{1} \cos \varphi_{2}\right)
\end{gathered}
$$

each point inside the image corresponds to two points on the integral surface $\mathcal{M}_{c, h}$, while each boundary point corresponds to one point. This consideration makes it easy to determine the type of integral surface. As can be seen in Fig. 6, there are three types of integral surfaces in the reduced system: the torus $\mathbb{T}^{2}$, the sphere $\mathbf{S}^{2}$ and the 3-handle sphere $\mathcal{M}_{3}^{2}$ (an orientable two-dimensional surface of genus 3). 


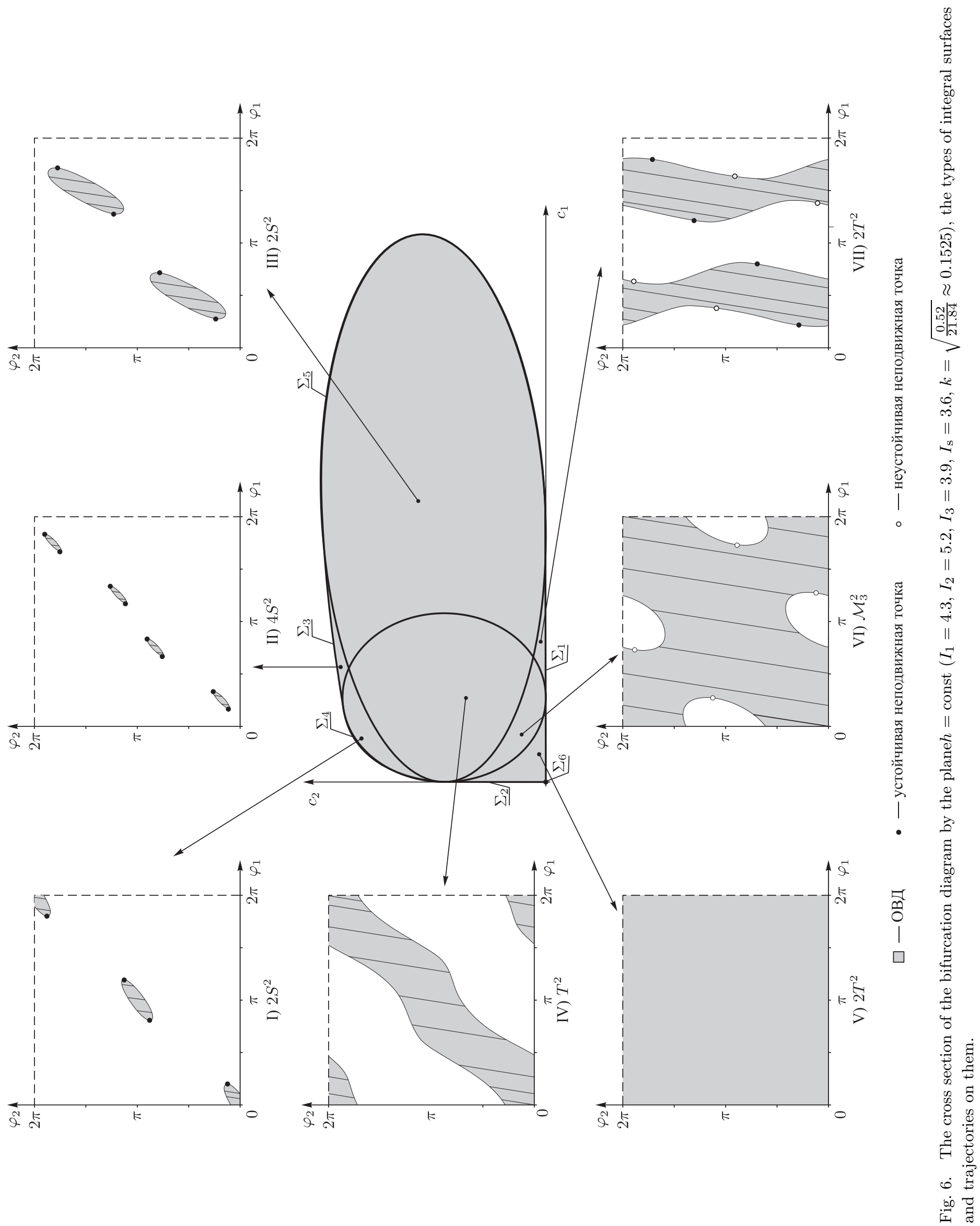


Comment. In this case, when the bifurcation curves are crossed, most of the bifurcations of the integral manifolds can be easily understood by using their projections on the plane $\left(\varphi_{1}, \varphi_{2}\right)$, see Fig. 6 . We will comment only on the bifurcations occurring at the intersection of the curves $\Sigma_{1}$ and $\Sigma_{2}$. As $\Sigma_{2}$ is approached, $c_{1} \rightarrow 0$ and, as follows from Eq. (6), the ellipse on the plane $\left(\omega_{1}, \omega_{2}\right)$ shrinks to a point, and each torus of the region $\mathrm{V}$ shrinks to a circle and then disappears. A similar bifurcation occurs at the intersection of $\Sigma_{1}$.

Classification of trajectories for $I_{3}<I_{2}<I_{1}$. We now proceed to determining the possible types of trajectories. As we have seen above (see Section 4), when projected on the torus $\left(\varphi_{1}, \varphi_{2}\right) \bmod 2 \pi$ the trajectories of the system are defined by the straight lines

$$
\varphi_{1}=k \varphi_{2}+c_{3}, \quad c_{3}=\text { const }, \quad k^{2}=\frac{\left(I_{1}-I_{3}\right)\left(I_{2}-I_{3}\right)}{I_{1} I_{2}} .
$$

Since $k$ does not depend on the constants of the first integrals, projections of the trajectories onto the torus $\left(\varphi_{1}, \varphi_{2}\right)$ are the same for all types of integral manifolds $\mathcal{M}_{c, h}^{2}$.

Among the integrable manifolds $\mathcal{M}_{c, h}^{2}$ we single out tori that are projected onto the entire square (more precisely, torus) $\left\{\left(\varphi_{1}, \varphi_{2}\right) \mid 0 \leqslant \varphi_{i} \leqslant 2 \pi\right\}$ and denote them as $\mathbb{T}_{\infty}^{2}$.

Proposition 4. If $k \in \mathbb{Q}$ (rational), then all nonsingular trajectories (i.e., those that do not have any equilibrium points in their closures) are closed, while if $k \notin \mathbb{Q}$ (irrational), then

- the trajectories are closed for all types of integral surfaces $\mathcal{M}_{c, h}^{2}$ other than the tori $\mathbb{T}_{\infty}^{2}$,

- the trajectories on the tori $\mathbb{T}_{\infty}^{2}$ in this case are quasiperiodic orbits.

Proof. The first and the last statements in this case are obvious, therefore we consider in more detail the situation where $k$ is irrational and the connected component $\mathcal{M}_{c, h}^{2}$ is not a torus. Consider the behavior of the system trajectory whose projection reaches a certain point on the boundary of the projection $\mathcal{M}_{c, h}^{2}$. According to Eq. (24), when we move in this orbit in the phase space, the sign of $\omega_{3}$ changes, and the point on the projection moves along the same trajectory but in the opposite direction. Since $k$ is irrational, the projection will sooner or later reach the boundary of $\mathcal{M}_{c, h}^{2}$ at exactly two points, that is, we will obtain a periodic solution.

The bifurcation diagram and integral surfaces for the case $I_{2}<I_{3}<I_{1}$. In this case, the images of families of critical points coincide with those found previously, except for the family $\Sigma_{1}$ :

$$
\Sigma_{1}=\left\{\left(c_{1}, 0, h\right) \mid \frac{I_{2}-I_{3}}{I_{2} I_{\mathrm{s}}+I_{3}^{2}} h \leqslant \frac{c_{1}}{2 I_{3}^{2}\left(I_{3}+I_{\mathrm{s}}\right)} \leqslant \frac{I_{1}-I_{3}}{I_{1} I_{\mathrm{s}}+I_{3}^{2}} h\right\} .
$$

The typical form of the bifurcation surfaces $\Sigma_{3}, \Sigma_{4}, \Sigma_{5}$ for the case at hand is shown in Fig. 7 .

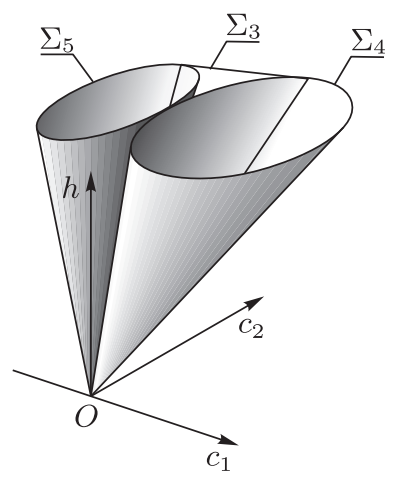

Fig. 7. The typical form of the bifurcation surfaces $\Sigma_{3}, \Sigma_{4}, \Sigma_{5}$ for the case $I_{2}<I_{3}<I_{1}\left(I_{1}=5.2, I_{2}=3.3, I_{3}=4, I_{\mathrm{S}}=3.6\right)$. 
As in the previous case, all sections of the bifurcation diagram formed by the intersection with the plane $h=$ const are similar to each other. Therefore, in what follows we consider the section of the threedimensional diagram formed by the intersection with the plane $h=$ const, the typical form of which is shown in Fig. 10.

As before, in order to determine the type of the integral surface $\mathcal{M}_{c, h}$ for each of the regions in the bifurcation diagram, we will construct its projection (Fig. 10). The main difference is that in this case $\varphi_{1}^{ \pm}$ is not an angle variable (see Section 4).

Since

$$
\begin{gathered}
\omega_{3}= \pm \sqrt{2 h-\frac{2 Q}{I_{3}^{2}\left(I_{3}+I_{\mathrm{s}}\right)}}, \\
Q=\frac{I_{\mathrm{s}} c_{2}}{2}+\frac{c_{1}}{2}\left(\frac{I_{\mathrm{s}} I_{1}+I_{3}^{2}}{I_{1}-I_{3}} \cosh ^{2} \varphi_{1}^{ \pm}-\frac{I_{\mathrm{s}} I_{2}+I_{3}^{2}}{I_{2}-I_{3}} \sinh ^{2} \varphi_{1}^{ \pm}\right)- \\
-I_{\mathrm{s}} \sqrt{c_{2}}\left(\sqrt{\frac{I_{1} c_{1}}{I_{1}-I_{3}}} \cosh \varphi_{1}^{ \pm} \sin \varphi_{2}+\sqrt{\frac{I_{2} c_{1}}{I_{3}-I_{2}}} \sinh \varphi_{1}^{ \pm} \cos \varphi_{2}\right)
\end{gathered}
$$

each point inside the image corresponds to two points on the integral surface $\mathcal{M}_{c, h}$, while each boundary point corresponds to one point. As can be seen in Fig. 10, there are two types of integral surfaces in the reduced system: the torus $\mathbb{T}^{2}$ and the sphere $\mathbf{S}^{2}$.

Comment. We will explain in more detail why in this case it is not the entire line segment $c_{1}=0$ (located inside the RPM), but only the portion from the origin to the point of contact of the ellipses $\Sigma_{4}$ and $\Sigma_{5}$ that corresponds to the bifurcation curve $\Sigma_{2}$.

In this case, the integral $C_{1}$ defines on the plane $\left(\omega_{1}, \omega_{2}\right)$ a family of curves of the form

$$
I_{1}\left(I_{1}-I_{3}\right) \omega_{1}^{2}-I_{2}\left(I_{3}-I_{2}\right) \omega_{2}^{2}=c_{1} .
$$

When $c_{1} \neq 0$, this relation defines a pair of hyperbolas, while when $c_{1}=0$, it defines a pair of intersecting straight lines (and the parametrization (20) does not work). When the sign of $c_{1}$ changes, these curves are deformed as follows:
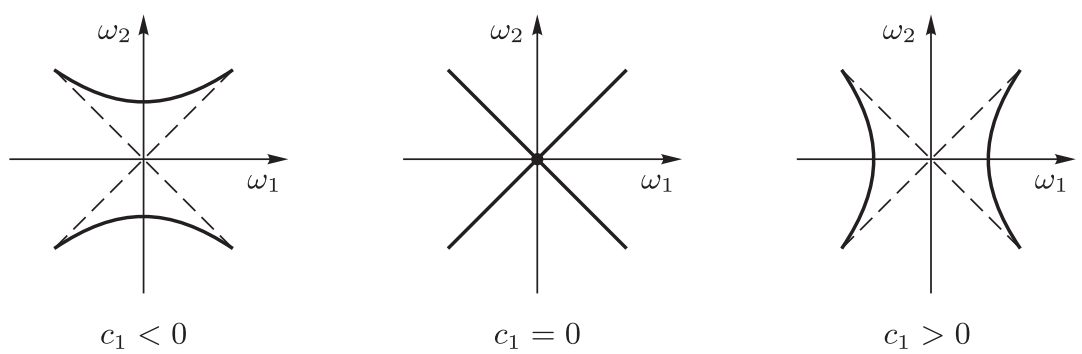

Fig. 8.

If the change of the sign of $c_{1}$ occurs in region I of the bifurcation diagram (i.e., above the point of contact of the curves $\Sigma_{4}$ and $\Sigma_{5}$ ), then the integral manifolds that are always located far from the origin $\omega_{1}=\omega_{2}=0$ are deformed without bifurcations when the sign changes.

If we cross the straight line $c_{1}=0$ in the direction from region IV to region $\mathrm{V}$ (or vice versa), then, as can be seen in Fig. 8, the projection of the integral manifold on the plane $\left(\omega_{1}, \omega_{2}\right)$ passes through the origin of the plane. After bifurcation, having passed through the singular manifold with a pair of critical circles, the integral manifold itself, consisting of a pair of tori, turns into a pair of tori. A similar bifurcation of the tori in the three-dimensional space can be schematically depicted as 


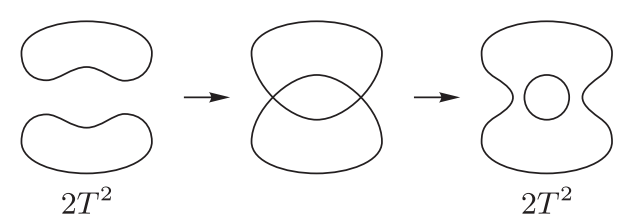

Fig. 9.

where these curves must be multiplied by $S^{1}$ to obtain the corresponding two-dimensional surfaces.

Classification of trajectories for $I_{2}<I_{3}<I_{1}$. We now proceed to determining the possible types of trajectories. As we have seen above (see Section 4), the trajectories of the system are defined by the straight lines

$$
\varphi_{1}^{ \pm}=k \varphi_{2}+c_{3}, \quad c_{3}=\mathrm{const}, \quad k^{2}=\frac{\left(I_{1}-I_{3}\right)\left(I_{3}-I_{2}\right)}{I_{1} I_{2}} .
$$

Proposition 5. In this case, all the nonsingular trajectories of the system are closed.

The proof of this proposition is almost a verbatim repetition of the proof of closure in Proposition 4 , therefore we will omit it.

\section{Absolute Dynamics ${ }^{2}$}

\subsection{General Equations for Orientation of the Body and the Shell}

Let us now consider the problem of describing the orientation of the inner body and the shell. For this, we define three coordinate systems:

- an inertial coordinate system $C x y z$ in which the center of mass is fixed,

- a moving coordinate system $C x_{1} x_{2} x_{3}$ rigidly attached to the inner body,

- a moving coordinate system $C x_{1} x_{2} x_{3}$ rigidly attached to the shell.

Since the angular velocities of the bodies were written above in terms of the moving axes $C x_{1} x_{2} x_{3}$, as rotation matrices describing the configuration of the system we choose:

- $\mathbf{Q} \in S O(3)$, the matrix of rotation of the fixed axes relative to the inner body, with projections of the fixed unit vectors on the axes $C x_{1} x_{2} x_{3}$ written in the columns,

- $\mathbf{S} \in S O(3)$, the matrix of rotation of the shell relative to the inner body, with projections of the unit vectors of the shell on the axes $C x_{1} x_{2} x_{3}$ written in the columns.

If we denote the coordinates of an arbitrary vector in each of the above systems as

$\boldsymbol{r}_{0}=(x, y, z)$ in the system $C x y z$,

$\boldsymbol{r}=\left(x_{1}, x_{2}, x_{3}\right)$ in the system $C x_{1} x_{2} x_{3}$,

$\boldsymbol{R}=\left(X_{1}, X_{2}, X_{3}\right)$ in the system $C X_{1} X_{2} X_{3}$,

then obviously

$$
\boldsymbol{r}=\mathrm{Q} \boldsymbol{r}_{0}=\mathrm{S} \boldsymbol{R} .
$$

The evolution of the matrices $\mathbf{Q}$ and $\mathbf{S}$ is governed by

$$
\begin{gathered}
\dot{\mathbf{Q}}=\widetilde{\boldsymbol{\omega}} \mathbf{Q}, \quad \dot{\mathbf{S}}=(\widetilde{\boldsymbol{\omega}}-\widetilde{\boldsymbol{\Omega}}) \mathbf{S}, \\
\widetilde{\boldsymbol{\omega}}=\left(\begin{array}{ccc}
0 & \omega_{3} & -\omega_{2} \\
-\omega_{3} & 0 & \omega_{1} \\
\omega_{2} & -\omega_{1} & 0
\end{array}\right), \quad \widetilde{\boldsymbol{\Omega}}=\left(\begin{array}{ccc}
0 & \Omega_{3} & -\Omega_{2} \\
-\Omega_{3} & 0 & \Omega_{1} \\
\Omega_{2} & -\Omega_{1} & 0
\end{array}\right),
\end{gathered}
$$

\footnotetext{
${ }^{2}$ As we have mentioned above (see Section 2), the term absolute dynamics is used solely for the sake of brevity and is not associated with the existence of an absolute coordinate system
} 


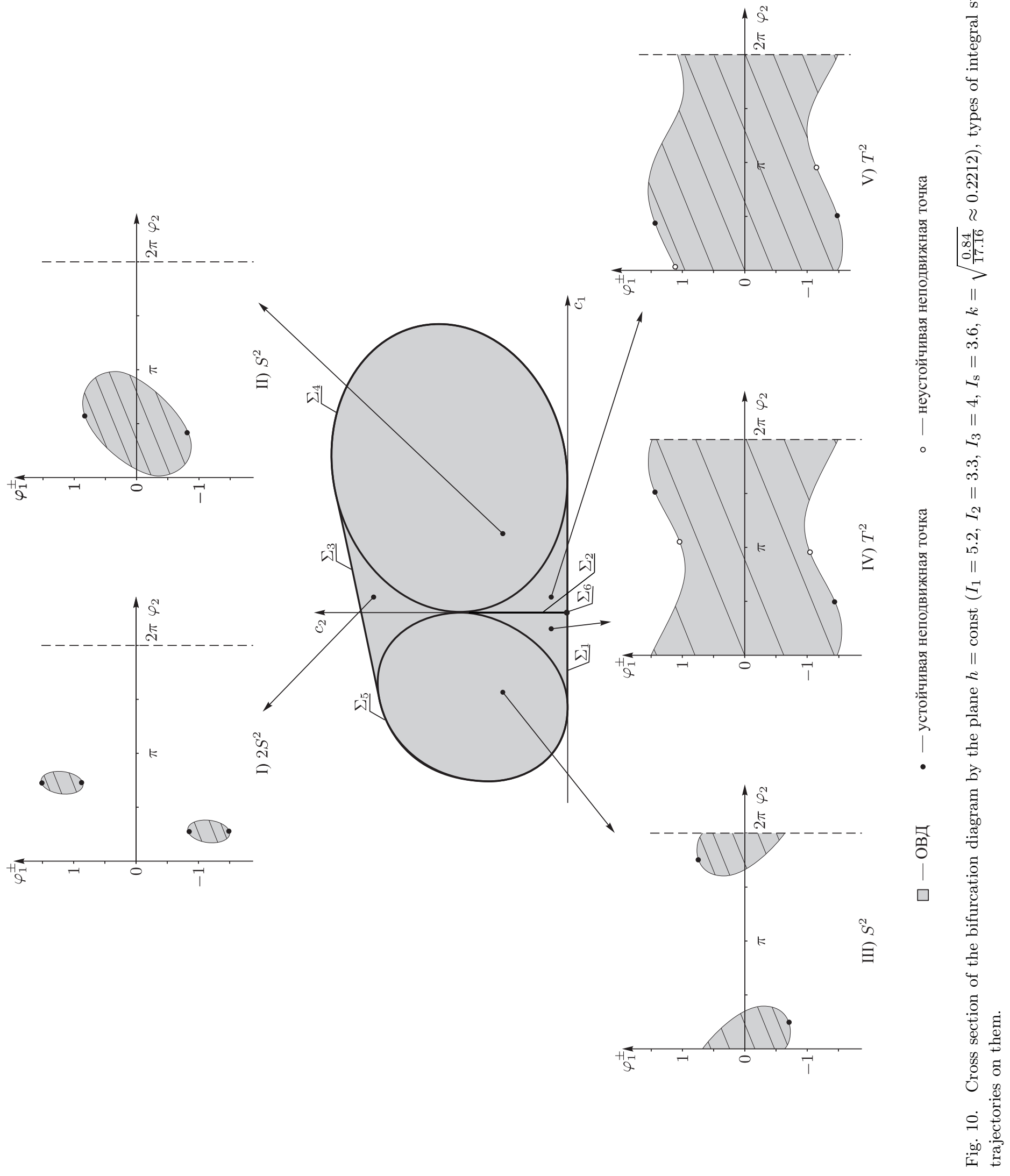


where $\omega_{i}$ and $\Omega_{i}$ are the projections of the absolute angular velocities of the body and the shell on the axes $O x_{1} x_{2} x_{3}$.

Remark 1. If we let $\boldsymbol{\alpha}, \boldsymbol{\beta}, \boldsymbol{\gamma}$ denote the columns of the matrix $\mathbf{Q}$, then in the vector form the matrix equation for $\mathbf{Q}$ will have the form of the well-known Poisson equations

$$
\dot{\alpha}=\alpha \times \omega, \quad \dot{\beta}=\beta \times \omega, \quad \dot{\gamma}=\gamma \times \omega .
$$

Thus, if $\boldsymbol{\omega}(t)$ and $\boldsymbol{\Omega}(t)$ are known, then to describe the configuration of the system it is necessary to integrate equations (27). We will not analyze these equations in the general case, but will only consider the problem of what kind of motion the body and the shell execute when the angular velocities $\boldsymbol{\omega}$ and $\boldsymbol{\Omega}$ define the fixed points in the system (5).

\subsection{Absolute Motion of Bodies with Constant $\omega$ and $\Omega$.}

To simplify the calculations, both the fixed axes $O x y z$ and the axes $O X_{1} X_{2} X_{3}$ rigidly attached to the shell can be chosen in an arbitrary manner (the choice of the latter is motivated by the spherical symmetry of the shell). First of all, note that the evolution of the angular momentum $\boldsymbol{M}=I_{\mathrm{S}} \boldsymbol{\Omega}+\mathbf{I} \boldsymbol{\omega}$ is governed by

$$
\dot{M}=\boldsymbol{M} \times \boldsymbol{\omega} .
$$

This implies that the momentum vector remains constant in the fixed axes. Using this, we choose

$$
C z \| M, \quad C x \perp M, \quad C y \perp M .
$$

Since all projections of $\omega_{i}$ and $\Omega_{i}$ remain constant for the fixed points of the system (5), the first matrix equation (27) can be integrated to give

$$
\mathbf{Q}=\left(\begin{array}{ccc}
\frac{\omega_{2}}{\nu_{1}} \sin \left(\nu_{1} t+\theta\right) & -\frac{\omega_{2}}{\nu_{1}} \cos \left(\nu_{1} t+\theta\right) & \frac{\omega_{1}}{\nu_{1}} \\
-\frac{\omega_{1}}{\nu_{1}} \sin \left(\nu_{1} t+\theta\right) & \frac{\omega_{1}}{\nu_{1}} \cos \left(\nu_{1} t+\theta\right) & \frac{\omega_{2}}{\nu_{1}} \\
-\cos \left(\nu_{1} t+\theta\right) & -\sin \left(\nu_{1} t+\theta\right) & 0
\end{array}\right),
$$

where $\nu_{1}=\sqrt{\omega_{1}^{2}+\omega_{2}^{2}}, \theta=$ const.

Similarly, we can integrate the equation for $\mathbf{S}$. To get it in the simplest form, we choose a coordinate system $C X_{1} X_{2} X_{3}$ attached to the shell in such a manner that at the initial moment of time it coincides with $C x_{1} x_{2} x_{3}$. We finally get

$$
\mathbf{S}=\left(\begin{array}{ccc}
\frac{\left(\Omega_{2}-\omega_{2}\right)^{2}}{\nu_{2}^{2}} \cos \left(\nu_{2} t\right)+\frac{\left(\Omega_{1}-\omega_{1}\right)^{2}}{\nu_{2}^{2}} & \frac{\left(\Omega_{1}-\omega_{1}\right)\left(\Omega_{2}-\omega_{2}\right)}{\nu_{2}^{2}}\left(1-\cos \left(\nu_{2} t\right)\right) & \frac{\Omega_{2}-\omega_{2}}{\nu_{2}} \sin \left(\nu_{2} t\right) \\
\frac{\left(\Omega_{1}-\omega_{1}\right)\left(\Omega_{2}-\omega_{2}\right)}{\nu_{2}^{2}}\left(1-\cos \left(\nu_{2} t\right)\right) & \frac{\left(\Omega_{1}-\omega_{1}\right)^{2}}{\nu_{2}^{2}} \cos \left(\nu_{2} t\right)+\frac{\left(\Omega_{2}-\omega_{2}\right)^{2}}{\nu_{2}^{2}} & -\frac{\Omega_{1}-\omega_{1}}{\nu_{2}} \sin \left(\nu_{2} t\right) \\
-\frac{\Omega_{2}-\omega_{2}}{\nu_{2}} \sin \left(\nu_{2} t\right) & \frac{\Omega_{1}-\omega_{1}}{\nu_{2}} \sin \left(\nu_{2} t\right) & \cos \left(\nu_{2} t\right)
\end{array}\right)
$$

where $\nu_{2}=\sqrt{\left(\Omega_{1}-\omega_{1}\right)^{2}+\left(\Omega_{2}-\omega_{2}\right)^{2}}$.

That is, when $\boldsymbol{\omega}$ and $\boldsymbol{\Omega}$ are constant, the system executes double-period motion, i.e., nonsingular trajectories in the phase space are periodic (if $\frac{\nu_{1}}{\nu_{2}} \in \mathbb{Q}$ ) and quasiperiodic (if $\frac{\nu_{1}}{\nu_{2}} \notin \mathbb{Q}$ ) orbits of twodimensional tori.

\section{Appendix.}

\section{Stability of Equilibrium Points in Hamiltonian Systems with a Rank-Two Poisson Structure}

Let $\mathbf{J}$ be a Poisson structure of rank 2 and $H$ be a Hamiltonian. How should the stability of equilibrium points be checked in this case on a symplectic leaf and on a Poisson manifold in general?

Note that a Poisson structure of rank 2 can be represented locally in a neighborhood of a regular point as $\boldsymbol{\xi} \wedge \boldsymbol{\eta}$, where $\boldsymbol{\xi}$ and $\boldsymbol{\eta}$ are some vector fields. Sometimes such a representation can be defined globally (then the Poisson structure is called decomposable (see [Konyaev, 2014]). But even if a global representation does not exist, it is not difficult to find it locally. Note that in order for the decomposable 
bivector $\boldsymbol{\xi} \wedge \boldsymbol{\eta}$ to define a Poisson structure, it is necessary and sufficient that (at regular points) the condition $[\boldsymbol{\xi}, \boldsymbol{\eta}]=f \boldsymbol{\xi}+g \boldsymbol{\eta}$ is satisfied for certain functions $f$ and $g$.

If such a decomposition of the Poisson structure is specified, then the question of stability is resolved by calculating the Hessian of the function $H$ restricted to a symplectic leaf. The following statement shows how this can be done without calculating the Casimir functions $C_{1}, \ldots, C_{k}$ and without explicitly imposing the restriction $\left.H\right|_{M_{c}}$, where $M_{c}=\left\{C_{i}=c_{i}, i=1,2 \ldots, k\right\}$.

Theorem 2. Let $x_{0}$ be an equilibrium point of a Hamiltonian system $\dot{\boldsymbol{x}}=\mathbf{J} d H(\boldsymbol{x})$ and

$$
\mu_{H}\left(\boldsymbol{x}_{0}\right)=\left|\begin{array}{ll}
\boldsymbol{\xi}(\boldsymbol{\xi}(H)) & \boldsymbol{\xi}(\boldsymbol{\eta}(H)) \\
\boldsymbol{\xi}(\boldsymbol{\eta}(H)) & \boldsymbol{\eta}(\boldsymbol{\eta}(H))
\end{array}\right|
$$

where $\boldsymbol{\xi}(H)$ is the derivative $H$ along the vector field $\boldsymbol{\xi}$ (the value of the determinant is taken at the point $\left.\boldsymbol{x}_{0}\right)$. Then

(1) if $\mu_{h}\left(\boldsymbol{x}_{0}\right)>0$, then $\boldsymbol{x}_{0}$ is a stable equilibrium point;

(2) if $\mu_{h}\left(\boldsymbol{x}_{0}\right)<0$, then $\boldsymbol{x}_{0}$ is an unstable equilibrium point.

Proof. In the neighborhood of $\boldsymbol{x}_{0}$, let us consider a local coordinate system $x_{1}, x_{2}, C_{1}, \ldots, C_{k}$, where $C_{1}, \ldots, C_{k}$ are the Casimir functions, and $x_{1}$ and $x_{2}$ are regarded as local coordinates on the symplectic leaf $M_{c}$ such that at the point $\boldsymbol{x}_{0}$ we have $\partial_{x_{1}}=\boldsymbol{\xi}, \partial_{x_{2}}=\boldsymbol{\eta}$ (it is clear that we can always ensure that this condition is satisfied at one distinguished point, making, if necessary, a linear change of variables).

Since the rank of $\mathbf{J}$ equals two, we can consider our Hamiltonian system as a system with one degree of freedom in which the Casimirs $C_{1}, \ldots, C_{k}$ play the role of parameters. The fact that $\boldsymbol{x}_{0}$ is the equilibrium point means that $\frac{\partial H}{\partial x_{1}}=\frac{\partial H}{\partial x_{2}}=0$. Its stability (both on an individual symplectic leaf and on the entire Poisson manifold) is guaranteed by the well-known inequality

$$
\operatorname{det} d^{2} H\left(\boldsymbol{x}_{0}\right)=\left|\begin{array}{cc}
\frac{\partial^{2} H}{\partial x_{1}^{2}}\left(\boldsymbol{x}_{0}\right) & \frac{\partial^{2} H}{\partial x_{1} \partial x_{2}}\left(\boldsymbol{x}_{0}\right) \\
\frac{\partial^{2} H}{\partial x_{1} \partial x_{2}}\left(\boldsymbol{x}_{0}\right) & \frac{\partial^{2} H}{\partial x_{2}^{2}}\left(\boldsymbol{x}_{0}\right)
\end{array}\right|>0 .
$$

Conversely, instability is guaranteed by the opposite (strict) inequality. Therefore, it suffices to verify that $\mu_{H}\left(\boldsymbol{x}_{0}\right)=\operatorname{det} d^{2} H\left(\boldsymbol{x}_{0}\right)$.

This fact is almost obvious. Indeed, since $\boldsymbol{\xi}$ and $\boldsymbol{\eta}$ are tangent to the symplectic leaves, we have

$$
\boldsymbol{\xi}=\xi_{1} \partial_{x_{1}}+\xi_{2} \partial_{x_{2}}, \quad \boldsymbol{\eta}=\eta_{1} \partial_{x_{1}}+\eta_{2} \partial_{x_{2}}
$$

where $\xi_{i}$ and $\eta_{i}$ are smooth functions such that $\xi_{1}\left(\boldsymbol{x}_{0}\right)=\eta_{2}\left(\boldsymbol{x}_{0}\right)=1, \xi_{2}\left(\boldsymbol{x}_{0}\right)=\eta_{1}\left(\boldsymbol{x}_{0}\right)=0$. Hence we obtain by direct calculation

$$
\boldsymbol{\xi}(\boldsymbol{\xi}(H))=\sum_{i, j=1}^{2} \frac{\partial^{2} H}{\partial x_{i} \partial x_{j}} \xi_{i} \xi_{j}+\left(\text { terms containing } \frac{\partial H}{\partial x_{1}} \text { and } \frac{\partial H}{\partial x_{2}}\right) .
$$

Since at the point under consideration $\frac{\partial H}{\partial x_{1}}\left(\boldsymbol{x}_{0}\right)=\frac{\partial H}{\partial x_{2}}\left(\boldsymbol{x}_{0}\right)=0$, the second term vanishes. Furthermore, due to the special selection of our coordinate system, we have $\xi_{1}\left(\boldsymbol{x}_{0}\right)=1, \xi_{2}\left(\boldsymbol{x}_{0}\right)=0$. This yields

$$
\left.\boldsymbol{\xi}(\boldsymbol{\xi}(H))\right|_{\boldsymbol{x}_{0}}=\frac{\partial^{2} H}{\partial x_{1}^{2}}\left(\boldsymbol{x}_{0}\right)
$$

Likewise, $\left.\boldsymbol{\eta}(\boldsymbol{\eta}(H))\right|_{\boldsymbol{x}_{0}}=\frac{\partial^{2} H}{\partial x_{2}^{2}}\left(\boldsymbol{x}_{0}\right)$ and $\left.\boldsymbol{\xi}(\boldsymbol{\eta}(H))\right|_{\boldsymbol{x}_{0}}=\frac{\partial^{2} H}{\partial x_{1} \partial x_{2}}\left(\boldsymbol{x}_{0}\right)$. In other words, $\mu_{H}\left(\boldsymbol{x}_{0}\right)=\operatorname{det} d^{2} H\left(\boldsymbol{x}_{0}\right)$, Q.E.D.

Remark 2. If $\mu_{h}\left(\boldsymbol{x}_{0}\right)=0$, then the question of stability requires further investigation. 


\section{Conclusion}

In conclusion, we mention some issues associated with this study and requiring further investigation. It would be interesting to understand the generality of the investigated integration procedure in nonholonomic mechanics, that is, to find new physically interesting problems that can be solved using this procedure. We note that there exist brackets of rank two of a somewhat different nature - chaotic Poisson brackets. There is not a single Casimir function for them, and the system that is defined on them may exhibit chaotic behavior. Such brackets occur in the well-known Suslov problem. It would be interesting to find out the mechanism of formation of such brackets and to identify additional mechanically substantial problems in which they are encountered.

\section{References}

Bizyaev I. A., Borisov A. V. \& Mamaev I. S. [2013] "The Dynamics of Nonholonomic Systems Consisting of a Spherical Shell with a Moving Rigid Body Inside," Rus. J. Nonlin. Dyn. 9, pp. 547-566 (Russian). (See also: [2014] Regul. Chaotic Dyn. 19, pp. 198-213.)

Bolsinov A. V., Izosimov A. M., Konyaev, A. Yu. \& Oshemkov, A. A. [2012] "Algebra and Topology of Integrable Systems. Research problems," Trudy Sem. Vektor. Tenzor. Anal. 28, pp. 119-191 (Russian).

Bolsinov A. V., Borisov A. V. \& Mamaev I. S. [2010] "Topology and Stability of Integrable Systems," Uspekhi Mat. Nauk 65, no. 2(392), pp. 71-132 (Russian).

Bolsinov A. V. \& Taimanov I. A. [2000a] "Integrable Geodesic Flows with Positive Topological Entropy," Inventiones mathematicae 140, pp. 639-650.

Bolsinov A. V. \& Taimanov I. A. [2000b] "Integral Geodesic Flows on Supsension Automorphisms of Tori," Trudy Mat. Inst. Steklov 231, pp. 46-63 (Russian).

Borisov A. V., Kilin A. A. \& Mamaev I. S. [2010] "Hamiltonicity and Integrability of the Suslov Problem," Rus. J. Nonlin. Dyn. 6, pp. 127-142 (Russian). (See also: [2011] Regul. Chaotic Dyn. 16, pp. 104-116.)

Borisov A. V. \& Mamaev I. S. [2002] "The Rolling of a Rigid Body on a Plane and Sphere: Hierarchy of Dynamics," Regul. Chaotic Dyn. 7, pp. 177-200. (See also: [2002] Nonholonomic Dynamical Systems, MoscowIzhevsk: Regul. Chaotic Dyn., Inst. Computer Science, pp. 173-205 (Russian).)

Borisov A. V. \& Mamaev I. S. [2008] "Conservation Laws, Hierarchy of Dynamics and Explicit Integration of Nonholonomic Systems," Rus. J. Nonlin. Dyn. 4, pp. 223-280 (Russian). (See also: [2008] Regul. Chaotic Dyn. 13, pp. 443-490.)

Borisov A. V., Mamaev I.S. \& Bizyaev I. A. [2013] "The Hierarchy of Dynamics of a Rigid Body Rolling without Slipping and Spinning on a Plane and a Sphere," Regul. Chaotic Dyn. 18, pp. 277-328. (See also: [2013] Rus. J. Nonlin. Dyn. 9, pp. 141-202 (Russian).)

Borisov A. V., Mamaev I. S. \& Kilin A. A. [2002] "The Rolling Motion of a Ball on a Surface: New Integrals and Hierarchy of Dynamics," Regul. Chaotic Dyn. 7, pp. 200-219. (See also: [2002] Nonholonomic Dynamical Systems, MoscowIzhevsk: Regul. Chaotic Dyn., Inst. Computer Science, pp. 206-226 (Russian).)

Butler L. [2003] "Integrable Geodesic Flows with Wild First Integrals: the Case of Two-Step Nilmanifolds", Ergodic Theory and Dynamical Systems 23, pp. 771-797.

Fuller F. B. [1971] "The Writhing Number of a Space Curve," Proc. Natl. Acad. Sci. USA 68, pp. 815-819.

Kharlamov A. P. \& Kharlamov M. P. [1995] "Nonholonomic Hinge," Ukrainian National Academy of Sciences, Mekhanika tverdogo tela, 27, pp. 1-7 (Russian).

Konyaev A. Yu. [2014] "Classification of Lie Algebras with Generic Orbits of Dimension 2 in the Coadjoint Representation," Sb. Math. 205, pp. 47-66 (Russian).

Kozlov V. V. \& Kolesnikov N. N. [1978] "On Theorems of Dynamics," J. Appl. Math. Mech. 42, pp. 28-33 (Russian).

Suslov G. K. [1946] Theoretical Mechanics (Moscow-Leningrad: Gostechizdat), 655 pages (Russian).

Wagner V. V. [1941] "The Geometrical Interpretation of the Motion of Nonholonomic Dynamical Systems," Trudy Seminara po Vekt. i Tenz. Anal. 5, pp. 301-327 (Russian). 